\title{
Temporal Dynamics and Response Modulation across the Human Visual System in a Spatial Attention Task: An ECoG Study
}

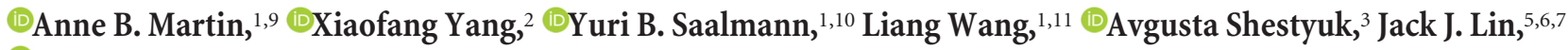 \\ - Josef Parvizi, ${ }^{8}$ Robert T. Knight, ${ }^{3,4}$ and Sabine Kastner ${ }^{1,2}$ \\ ${ }^{1}$ Princeton Neuroscience Institute, ${ }^{2}$ Department of Psychology, Princeton University, Princeton, New Jersey 08544, ${ }^{3}$ Helen Wills Neuroscience Institute, \\ ${ }^{4}$ Department of Psychology, University of California, Berkeley, Berkeley, California 94720, ${ }^{5}$ Center for the Neurobiology of Learning and Memory, \\ ${ }^{6}$ Department of Biomedical Engineering, ${ }^{7}$ Department of Neurology, University of California, Irvine, Orange, California 92868 , ${ }^{8}$ Department of Neurology \\ and Neurological Sciences, Stanford University, Palo Alto, California 94304, ${ }^{9}$ Division of Biology and Biological Engineering, California Institute of \\ Technology, Pasadena, California 91125, ${ }^{10}$ Department of Psychology, University of Wisconsin-Madison, Madison, Wisconsin 53706, and ${ }^{11}$ CAS Key \\ Laboratory of Mental Health, Institute of Psychology, Chinese Academy of Sciences, Beijing 100101, China
}

The selection of behaviorally relevant information from cluttered visual scenes (often referred to as "attention") is mediated by a cortical large-scale network consisting of areas in occipital, temporal, parietal, and frontal cortex that is organized into a functional hierarchy of feedforward and feedback pathways. In the human brain, little is known about the temporal dynamics of attentional processing from studies at the mesoscopic level of electrocorticography $(\mathrm{ECoG})$, that combines millisecond temporal resolution with precise anatomical localization of recording sites. We analyzed high-frequency broadband responses (HFB) responses from 626 electrodes implanted in 8 epilepsy patients who performed a spatial attention task. Electrode locations were reconstructed using a probabilistic atlas of the human visual system. HFB responses showed high spatial selectivity and tuning, constituting ECoG response fields (RFs), within and outside the topographic visual system. In accordance with monkey physiology studies, both RF widths and onset latencies increased systematically across the visual processing hierarchy. We used the spatial specificity of HFB responses to quantitatively study spatial attention effects and their temporal dynamics to probe a hierarchical top-down model suggesting that feedback signals back propagate the visual processing hierarchy. Consistent with such a model, the strengths of attentional modulation were found to be greater and modulation latencies to be shorter in posterior parietal cortex, middle temporal cortex and ventral extrastriate cortex compared with early visual cortex. However, inconsistent with such a model, attention effects were weaker and more delayed in anterior parietal and frontal cortex.

Key words: attention latencies; onset latencies; spatial response fields; topographic atlas

Significance Statement

In the human brain, visual attention has been predominantly studied using methods with high spatial, but poor temporal resolution such as fMRI, or high temporal, but poor spatial resolution such as EEG/MEG. Here, we investigate temporal dynamics and attention effects across the human visual system at a mesoscopic level that combines precise spatial and temporal measurements by using electrocorticography in epilepsy patients performing a classical spatial attention task. Electrode locations were reconstructed using a probabilistic atlas of the human visual system, thereby relating them to topography and processing hierarchy. We demonstrate regional differences in temporal dynamics across the attention network. Our findings do not fully support a topdown model that promotes influences on visual cortex by reversing the processing hierarchy.

\section{Introduction}

The selection of information from cluttered visual environments (often referred to as "attention") is a fundamental problem in cognitive neuroscience. This process is mediated by a cortical

Received July 23, 2018; revised Oct. 15, 2018; accepted Nov. 6, 2018.

Author contributions: A.B.M., X.Y., Y.B.S., L.W., A.S., J.J.L., J.P., R.T.K., and S.K. edited the paper; Y.B.S. and S.K. designed research; A.S., J.J.L., J.P., and R.T.K. performed research; A.B.M., X.Y., and L.W. analyzed data; A.B.M., X.Y., and S.K. wrote the paper. large-scale network consisting of areas in occipital, temporal, parietal, and frontal cortex (Desimone and Duncan, 1995; Kastner and Ungerleider, 2000; Corbetta and Shulman, 2002; Saalmann and Kastner, 2011; Buschman and Kastner, 2015; Caspari et al., 
2015; Moore and Zirnsak, 2017). Anatomical and functional studies indicate that this network is organized into a hierarchy of feedforward and feedback pathways that are dynamically modulated by attention for selective routing of information. Anatomically, this processing hierarchy is constrained by specific laminar projection patterns that index feedforward and feedback connectivity (Felleman and Van Essen, 1991; Markov et al., 2014). Functionally, it is characterized by inter-areal interactions that use distinct frequency channels indexing feedforward and feedback signaling (van Kerkoerle et al., 2014; Bastos et al., 2015; Michalareas et al., 2016). Evidence from studies in patients suffering from attentional deficits because of brain damage, as well as inactivation and microstimulation studies in nonhuman primates, indicate that frontoparietal areas generate attention-related modulatory signals that are fed back to sensory cortex (Barceló et al., 2000; Moore and Armstrong, 2003; Corbetta and Shulman, 2011). Consistent with such a feedback model of attention control, it has been shown in monkey physiology studies that modulatory attention effects are greater and modulation latencies are shorter in higher-order compared with lower-order cortex, suggesting that attention-related feedback signals reverse the visual processing hierarchy (Mehta et al., 2000; Buffalo et al., 2010).

In the human brain, selective attention has been predominantly studied with methods that emphasize network level analyses and have either relatively high spatial, but poor temporal resolution such as fMRI, or high temporal, but relatively poor spatial resolution such as MEG/EEG. The functional hierarchy of feedforward and feedback pathways based on inter-areal interactions has been recently reported for the human visual system using MEG (Michalareas et al., 2016). However, the precise temporal dynamics during feedforward and feedback selective visual processing are not known. Only few studies have been performed at the mesoscopic level of intracranial EEG, or electrocorticography (ECoG), that combines millisecond temporal resolution with precise anatomical localization of recording sites (for review, see Parvizi and Kastner, 2018). In particular, highfrequency broadband (HFB) responses $>70 \mathrm{~Hz}$ show timelocking to specific sensory, motor, and cognitive events (Kreiman et al., 2006; Flinker et al., 2011; Hermes et al., 2012; Mesgarani et al., 2014). Thus far, spatially and feature-specific attentional modulation of HFB responses have been reported in visual cortex (Yoshor et al., 2007; Davidesco et al., 2013; Szczepanski et al., 2014).

Here, we studied HFB responses from hundreds of electrodes covering occipital, temporal, parietal, and frontal cortex in patients performing a classical spatial attention task. Electrode locations were reconstructed using a probabilistic atlas of the human visual system (Wang et al., 2015), thereby relating them to topography and processing hierarchy. We characterized the spatial specificity of HFB responses and used this property to quantitatively study spatial attention effects on baseline and visually-evoked activity across topographic and nontopographic cortex. Further, we investigated response onset and attentional modulation latencies to characterize the temporal dynamics of feedforward and feedback processing across the visual system during spatial attention.

Cognition, collaborative Grant (S.K. and R.T.K.). We thank Michael Arcaro for help with implementing the probabilistic atlas.

The authors declare no competing financial interests.

Correspondence should be addressed to Dr. Sabine Kastner, Princeton Neuroscience Institute and Department of Psychology, Washington Road, Princeton, NJ 08544. E-mail: skastner@princeton.edu.

https://doi.org/10.1523/JNEUROSCl.1889-18.2018

Copyright $\odot 2019$ the authors $\quad 0270-6474 / 19 / 390334-20 \$ 15.00 / 0$

\section{Table 1. Patient information}

\begin{tabular}{|c|c|c|c|c|c|c|c|c|c|}
\hline Subject & Sex & Age & Handec & Sovera & $\begin{array}{l}\text { Brain } \\
\text { areas }\end{array}$ & $\begin{array}{l}\text { No. of } \\
\text { electrodes }\end{array}$ & $\begin{array}{l}\text { Acc, } \\
\%\end{array}$ & $\mathrm{RT}, \mathrm{ms}$ & $\begin{array}{l}\text { No. of } \\
\text { trials }\end{array}$ \\
\hline 51 & $M$ & 45 & Right & RH & $0, P, T$ & 99 (112) & 96 & $802 \pm 13$ & 200 \\
\hline 52 & $M$ & 22 & Left & LH & $0, P, T$ & 110 (128) & 95 & 765 & 300 \\
\hline S3 & $\mathrm{F}$ & 22 & Right & LH & $\mathrm{LF}, 0, \mathrm{P}, \mathrm{MF}$ & $86(100)$ & 83 & $888 \pm 17$ & 200 \\
\hline$S 4^{\dagger}$ & M & 18 & Right & LH & $0, P, T$ & 86 (94) & $87^{\dagger}$ & $1024 \pm 16^{\dagger}$ & 200 \\
\hline$S 5^{*}$ & M & 42 & Left & LH & $L F, 0, P, T$ & 52 (74) & $97^{*}$ & $599 \pm 5^{*}$ & 300 \\
\hline S6 & M & 51 & Right & LH & $\mathrm{LF}, \mathrm{P}, \mathrm{MF}$ & $89(106)$ & $96 \quad 1$ & $1130 \pm 15$ & 250 \\
\hline S7 & M & 23 & Right & LH & $\mathrm{LF}, \mathrm{P}, \mathrm{T}$ & $52(52)$ & 98 & $794 \pm 11$ & 250 \\
\hline $\mathrm{S} 8^{+}$ & $\mathrm{F}$ & 56 & Right & $\mathrm{LH}$ & $L F, T$ & $62(64)$ & $91^{\dagger}$ & $955 \pm 19^{\dagger}$ & 150 \\
\hline
\end{tabular}

Area coverage by lobe: 0 , occipital; $P$, parietal; $T$, temporal; $L F$, lateral frontal; $M F$, medial frontal. The number of electrodes indicates those that were included in the analysis relative to all implanted electrodes (in parentheses). Subjects were tested with cues presented at ${ }^{*} 8$ or $\$ 16$ locations, respectively. All other subjects had cues presented at 14 locations. RH, Right hemisphere; LH, left hemisphere. ${ }^{1}$

\section{Materials and Methods}

\section{Subjects}

Eight subjects (S1-S8, 6 males, age: $35 \pm 5$, mean \pm SEM; for further information, see Table 1), who underwent presurgical epilepsy evaluation, provided written informed consent to participate in the study. Experimental procedures were approved by the Institutional Review Boards of the participating institutions. Anti-epileptic medications were discontinued for 2-3 d before testing, and subjects were seizure free for at least $5 \mathrm{~h}$ before testing. Subjects had normal or corrected-to-normal vision.

Subjects were implanted with 52-128 electrodes $(1 \mathrm{~cm}$ spacing in grids and strips), covering extensive parts of frontal, parietal, occipital, and temporal cortex in their left (7 subjects) and right ( 1 subject) hemispheres (for electrode locations from all subjects, see Fig. 1; for coverage information of each subject, see Table 1). The positioning of electrode grids and strips was entirely based on clinical criteria pertaining to diagnostic procedures.

\section{Visual display, stimuli, and task}

Visual displays were generated on a Dell Precision M4600 laptop (Dell) using Presentation software (Neurobehavioral Systems). Light gray stimuli were presented on a darker gray background at 50\% contrast (Fig. $2 A$ ). The timing of visual and auditory stimulus presentations was verified using a custom photodiode and microphone system. A microphone recorded auditory cues (starting tone and response feedback sounds; see next paragraph for task description). A photodiode placed at the lower right corner of the monitor recorded timing of each visual stimulus using a simultaneous light square presented at the location of the photodiode receptor. The computer screen was placed at a distance of $\sim 80 \mathrm{~cm}$ from the subject's eyes.

Subjects performed a variant of the Eriksen flanker task (Eriksen and Eriksen, 1974; Eriksen, 1995; Saalmann et al., 2012), discriminating between one of two target shapes that were shown embedded in a circular array of distracter shapes (Fig. 2A). Subjects were instructed to maintain fixation throughout the duration of each trial. Following a $2 \mathrm{~s}$ intertrial interval, each trial started with the presentation of a central fixation point $\left(0.5^{\circ}\right)$ and a coincidental tone. After $1100 \mathrm{~ms}$, a circular spatial cue $\left(1.5^{\circ}\right)$ was displayed for $100 \mathrm{~ms}$ at a pseudorandomly chosen peripheral location ( $7^{\circ}$ eccentricity), followed by a variable delay period (300-700 ms) and the presentation of a circular array of equally spaced barrel and bowtie shapes (each $\sim 2 \times 2^{\circ}$ ). The array was displayed for $2000 \mathrm{~ms}$ or until the subject responded, indicating with a left or right mouse-button press, respectively, whether a barrel or bowtie shape was presented at the cued location. Barrel and bowtie target stimuli were presented randomly with equal likelihood, and flanking shapes were either congruent (same shape in nearest neighboring positions) or incongruent (different shape in nearest neighboring positions). Feedback on performance was given to the subject upon completion of each trial via tones signaling a correct or incorrect response. To minimize stress for the patients, they were instructed to emphasize accuracy rather than speed of responses. Following task instructions, subjects performed a training block to familiarize themselves with the task. During the experiment, trials were presented in blocks of 50, and 3-6 blocks were recorded per subject (Table 1). The 

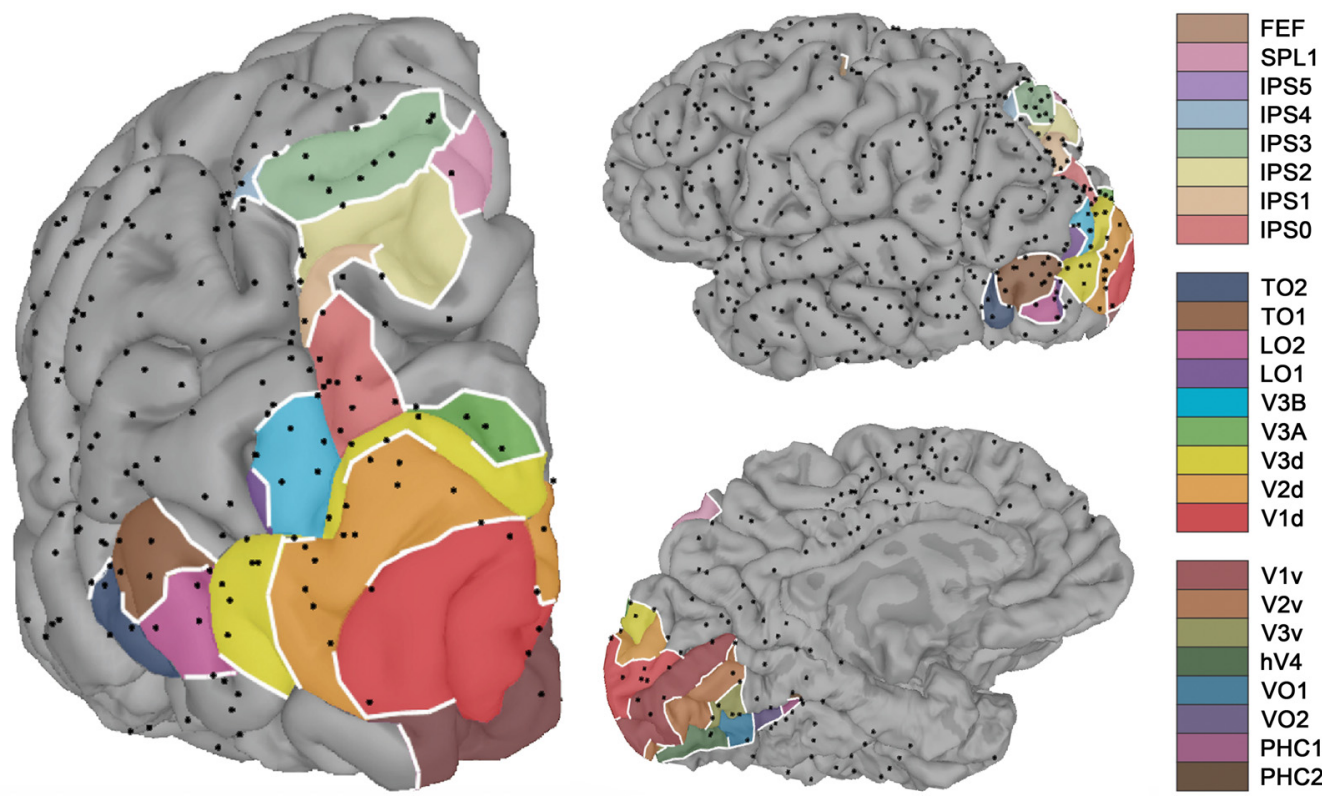

Figure 1. Electrode coverage. Electrode locations (combined across 8 patients; $N=636$ ) rendered onto a brain surface in standardized space, shown from posterior, lateral, and medial views. A probabilistic atlas of visuospatial topographic areas (Wang et al., 2015) is superimposed to clarify electrode locations relative to retinotopically organized cortex (see color-legend for areas on the right). Individual subject's electrodes were localized on their brain surfaces after aligning postoperative CT images of the implanted electrodes with preoperative structural MRIs; the surfaces and electrode locations were converted to a standard surface template. The probabilistic atlas was superimposed, and electrodes that overlapped the atlas maximum probability map were assigned to the maximally probable area (see Materials and Methods for further details).

A

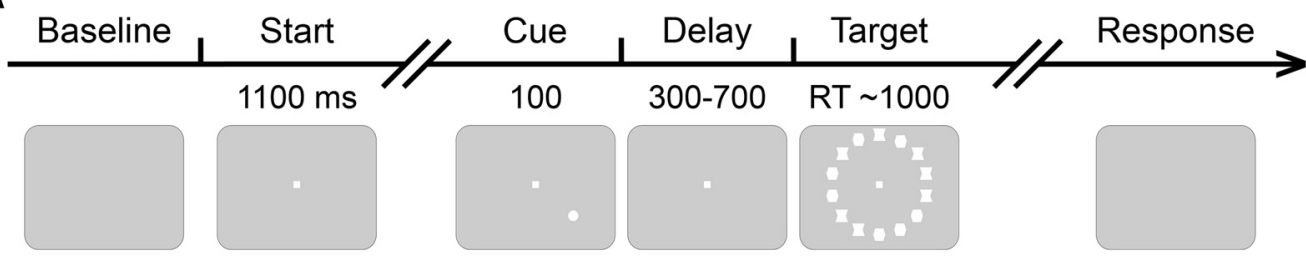

B

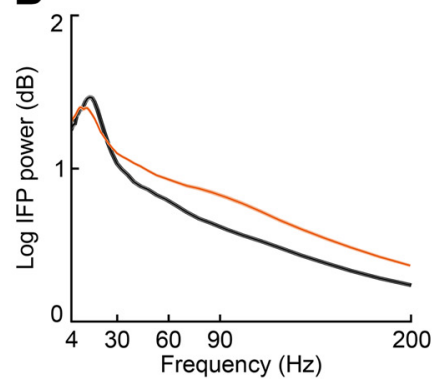

C

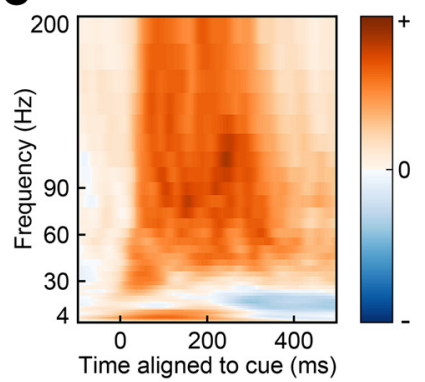

D

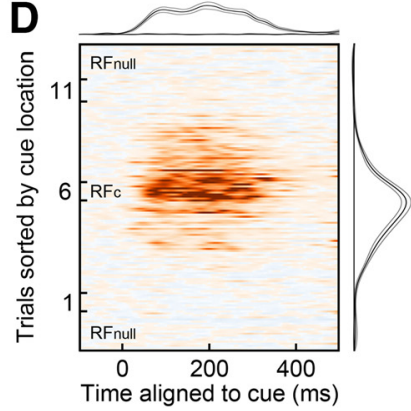

Figure 2. Task and example responses. $\boldsymbol{A}$, Subjects performed a variant of the Eriksen flanker task. After maintaining central fixation for $1100 \mathrm{~ms}$, a brief cue indicated the location of a target shape, which was displayed in a circular array after a variable delay of 300-700 ms. Targets were either barrel or bow-tie shapes, and flanking stimuli were either congruent (same shape) or incongruent (different shape). Subjects responded to indicate the target shape using a left or right mouse-button press. $\boldsymbol{B}$, IFP power recorded from one electrode located in area V3d as a function of frequency during baseline (black line; $200 \mathrm{~ms}$ before cue onset) and visually-evoked (orange line; $50-250 \mathrm{~ms}$ after cue onset) windows, mean \pm SEM across trials ( $N=149$ ). C, Relative cue-evoked IFP power ( $d^{\prime}$ of baseline), mean across trials at the location exerting the strongest HFB response (same electrode as in $\boldsymbol{B}$ ). Time 0 denotes cue onset. Color scale: \pm 2.5 . $\boldsymbol{D}$, Mean cue-evoked HFB power ( $70-200 \mathrm{~Hz}$, $d^{\prime}$ of baseline) at each cue location on a trial-by-trial basis (same electrode as in $\boldsymbol{B}$ and $\boldsymbol{C}$ ). Trials were sorted as a function of distance from the location exerting the strongest HFB response $\left(\mathrm{RF}_{\mathrm{C}}\right)$. Colors cale: \pm 30 . Top, Mean normalized power over time at $\mathrm{RF}_{\mathrm{C}} \pm$ trialwise SEM. Right, Mean normalized cue-evoked power at each cue location \pm trialwise $\mathrm{SEM}$.

number of cued locations and shapes in the target array was 8 ( 1 subject), 14 (5 subjects), or 16 ( 2 subjects).

To confirm fixation performance throughout the task, eye movements were visually monitored by the experimenter, and video recordings of the patient's face and eyes were performed throughout the experiment in the epilepsy monitoring care unit. No systematic saccadic eye movements were observed during task performance.

\section{Data acquisition}

Electrophysiological and peripheral (photodiode and microphone) channels were recorded using a 128-channel Tucker-Davis Technologies recording system at Stanford, a 128-channel Stellate Harmonic or Blackrock recording system at Johns Hopkins, a 128-channel Nihon Kohden recording system at Children's Hospital, and a 256-channel Nihon Kohden recording system (model JE120A) at UC Irvine. Signals were sam- 
pled at $3052 \mathrm{~Hz}$ (Tucker-Davis), $1000 \mathrm{~Hz}$ (Stellate), $5000 \mathrm{~Hz}$ (Nihon Kohden), or $10,000 \mathrm{~Hz}$ (Blackrock), amplified and filtered $(0.5-300 \mathrm{~Hz}$ at Stanford; $0.1-350 \mathrm{~Hz}$ (Stellate), or $0.3-2500 \mathrm{~Hz}$ (Blackrock, at Johns Hopkins), using a subdural electrode reference and a scalp ground. Data were digitized and resampled off-line at $1000 \mathrm{~Hz}$ to equate analysis across sites.

\section{Electrode localization}

For subjects S1-S6, postoperative CT images of the implanted electrodes were aligned with preoperative structural MRIs. For localization of electrodes within the visual system, a probabilistic atlas of visuospatial topographic areas, which is based on fMRI retinotopic mapping data from 53 healthy subjects (Wang et al., 2015), was combined with each subject's structural MRI. Specifically, after obtaining coregistration parameters between the MRI and CT images using normalized mutual information algorithms implemented in Bioimage Suite software, electrode locations were mapped onto a rendering of the 3 -D brain surface that was generated from the subject's structural MRI volume using FreeSurfer software (Dale et al., 1999; Fischl et al., 1999) and converted to a standard surface template using SUMA (Saad et al., 2004) and AFNI software. The probabilistic atlas of visuospatial topographic areas (Wang et al., 2015) was then superimposed onto each subject's brain surface. Using the maximum probability map, which assigns each node in the standard space to the topographic area with the highest probability, each electrode location that overlapped with the atlas was assigned to its maximally probable area. Sites that did not overlap the maximum probability map but were within one grid spacing $(N=17,10 \mathrm{~mm}$ spacing) to the nearest maximally probable area were included with the area. For subjects S7 and S8, the electrode locations were reconstructed on a standard surface based on postoperative drawings of the electrode positions. The electrode grids in these two subjects did not overlap with the probabilistic atlas. Recording sites outside visuospatial topographic areas were located using the Harvard-Oxford cortical parcellation that is based on anatomical markers (Desikan et al., 2006).

\section{Data analysis}

Behavioral data. For each subject, accuracy (as the proportion of correct trials relative to the number of all trials) and mean reaction times (RTs; averaged across all correct trials) were computed. Trials with RTs $>3 \mathrm{SD}$ from the mean were excluded from analyses (median $2 \%$ of trials, min $=$ $0.5 \%, \max =3.5 \%$ ). We also computed accuracy as a function of flanker condition to determine behavioral flanker effects (i.e., higher accuracy for congruent than incongruent conditions). Because response speed was not emphasized in our task, RTs were not a reliable measure of flanker effects. For the analyses of neural data, only trials with correct responses and appropriate RTs were included; there were insufficient numbers of incorrect trials for reliable analysis.

Neural data: preprocessing and time frequency analysis. A neurologist manually inspected all ECoG channels to identify those with interictal or ictal epileptiform activity and artifacts. Channels and epochs contaminated by epileptiform activity or abnormal signals (e.g., poor contact, excess drift, high-frequency noise) as well as those located over MRI defined abnormal sites were excluded from analysis (Table 1 shows the number of electrodes recorded and analyzed per subject). We excluded $16 \%$ of recorded electrodes based on these criteria (122/758). Off-line, the intracranial field potentials (IFPs) from the remaining 636 electrodes recorded across the eight subjects were referenced to each subject's common average. Power line noise and its harmonics were removed using a two-way zero phase-lag finite impulse response notch filter $( \pm 2 \mathrm{~Hz})$.

All analyses were performed using the EEGLAB toolbox (Delorme and Makeig, 2004) and customized scripts written in MATLAB (MathWorks). Time series were aligned separately to the cue and array onset and sorted by cue location. To increase the number of trials available for each analysis, trials from each cue location were combined with the two closest locations on either side (only in cases of 14-16 cue locations). This resulted in spatial smoothing around each location of $\sim 25^{\circ}$ of visual angle, yielding a minimum of 25 correct trials per cue location.

For each electrode, power spectra were calculated by applying a Hilbert transform to bandpass filtered ECoG IFPs. First, the IFPs were fil- tered using a two-way zero phase-lag finite impulse response filter. We defined the filter order as $3 r$, where $r$ is the ratio of the sampling rate to the low-frequency cutoff of the filter, rounded down, in each of the analyzed pass bands. For full-spectrum analyses, we used multiple logarithmicallyspaced pass bands with partially overlapping bands from $0.5-250 \mathrm{~Hz}$ (as by Voytek et al., 2013): the first pass band was seeded such that $f_{\mathrm{p}(1)}=$ $(0.5,0.9)$, and in subsequent bands $f_{\mathrm{L}(n)}=0.85 \times\left(f_{\mathrm{H}(n-1)}\right)$ and $f_{\mathrm{H}(n)}=$ $1.1 \times\left(f_{\mathrm{H}(n-1)}-f_{\mathrm{L}(n-1)}\right)+f_{\mathrm{L}(n)}$. We applied the Hilbert transform to each filtered time series $x$ to acquire the analytic amplitude $a_{x}(n)$. The instantaneous power in band $f_{\mathrm{p}(n)}$ at each time point in $x$ is the mean over trials of $a_{x}(n)$. In this paper, we focus our analyses on task-related power modulations in HFB responses $>70 \mathrm{~Hz}$ because of their high spatial specificity and temporal precision (Crone et al., 1998, 2006; Cheung et al., 2016; Parvizi and Kastner, 2018). Although the neural basis of HFB responses is still not entirely clear, these signals have been shown to correlate with multiunit activity obtained from thousands of neurons in the immediate vicinity of the recording electrode (Ray et al., 2008a; Ray and Maunsell, 2011; Rich and Wallis, 2017; Watson et al., 2018). More recent findings indicate $\mathrm{CA}+$ dendritic spikes in supragranular cortex as a principle contributor to pial HFB responses (Leszczyñski et al., Unpublished observations). Here, HFB responses were defined as the average power between the pass bands centered at 70 and $200 \mathrm{~Hz}$. These band definitions applied to the logarithmically-spaced bands yielded averages between 61.6 and $206.6 \mathrm{~Hz}$.

Outlier time points (HFB power modulations $>6 \mathrm{SD}$ of the mean for time points in the 50-400 ms following cue and array onset), and trials with outlier cue- or array-evoked power compared with other trials of that same condition (each trial mean in the interval 50-300 ms following the cue or array $>6 \mathrm{SD}$ of the mean across all trials in that condition) were eliminated. Typically, $<6 \%$ of trials per electrode were excluded $($ median $5 \%, \min =0 \%, \max =16 \%)$.

Identification of task-related activity. For each electrode, the mean IFP HFB power was calculated for each of the $8-16$ peripheral locations and for four task-related epochs: cue-evoked (50-250 ms after cue onset), delay-related (200 ms before array onset), early array-evoked (50-200 $\mathrm{ms}$ after array onset), and late array-evoked (300-500 ms after array onset). HFB power fluctuations during these epochs were compared with baseline activity occurring $200 \mathrm{~ms}$ before cue onset. Because there is no sharp transition in the signals between cue-evoked and delay activity, we defined the length of the presumed cue-evoked time interval post hoc based on the time course of cue-evoked activity in topographic area $\mathrm{V} 1 \mathrm{~d} / \mathrm{v}$, which showed a sharp decline of cue-evoked responses after 250 $\mathrm{ms}$ and did not appear to show any elevated delay activity in our recordings (see Fig. 4A, red trace). To avoid contamination of cue-evoked (i.e., sensory-driven) with delay-related (i.e., driven by the cognitive state) activity, only trials with delays $>450 \mathrm{~ms}$ (the median split of trials) were used for all analyses regarding delay-related activity. Similarly, to avoid contamination from motor responses, trials with reaction times $<500$ $\mathrm{ms}$ were excluded from analyses of array-related activity (median 0 , $\min =0, \max =9)$.

Task-responsive recording sites were identified based on the following criteria. First, a nonparametric cluster method (see Tests of Statistical Significance) was used to determine whether significant cue-evoked HFB power (compared with baseline) was sustained for at least 100 consecutive milliseconds at any of the peripheral locations. Second, the reliability of the trial-wise power at those locations was measured by generating bootstrapped distributions of the mean power during the cue-related epoch (1000 resamplings over trials of the cue-evoked HFB power relative to the mean baseline power); sites were included only if the $95 \%$ confidence interval (CI) of the bootstrapped distribution was greater than zero. Sites with significant delay- or array-related HFB power modulation were identified using the second criterion applied to the respective epochs.

Spatial tuning functions. After identifying sites with significant taskevoked responses in the HFB power of the IFPs for at least one peripheral location, we examined their relative responses across all peripheral locations to determine their spatial tuning properties. In cases of spatial tuning, we defined a response field center $\left(\mathrm{RF}_{\mathrm{C}}\right)$ as the location evoking the strongest power relative to baseline in response to the cue. Each site 

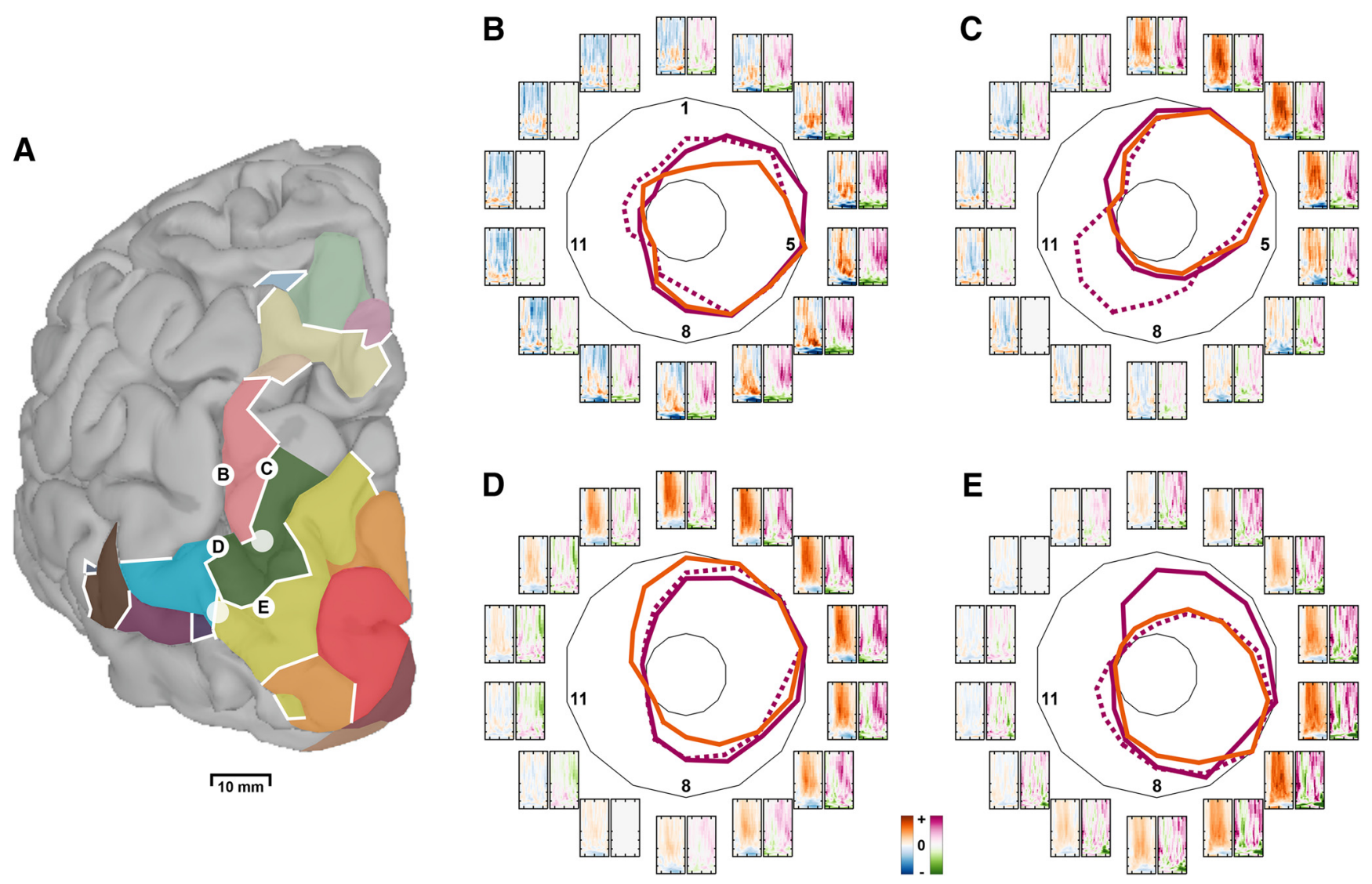

Figure 3. Spatial specificity of task-related HFB response fields. Recordings from four adjacent electrodes in subject $S 3$ ( $10 \mathrm{~mm}$ strip spacing). $\boldsymbol{A}$, Electrode positions projected on the subject's anatomical MRI surface in relation to the probabilistic atlas (color-coded atlas legend as in Fig. 1). Electrode diameters are shown to scale. Blank circles indicate electrodes in the strip lacking response fields. $\boldsymbol{B}-\boldsymbol{E}$, IFP power spectra (mean across trials) evoked by the cue (left) and array (right) at each of the 14 cue locations. Cue-evoked power is shown as $d^{\prime}$ of baseline power, indicating visually-evoked responses. Array-evoked power is shown as $d^{\prime}$ of power at $\mathrm{RF}_{\text {null, }}$ indicating the effect of spatial attention. Central panels, Circular tuning curves showing normalized $\mathrm{HFB}$ power at each location evoked by the cue (mean of $50-250 \mathrm{~ms}$ after cue, solid orange line), during the delay (mean of $200 \mathrm{~ms}$ before array, dashed purple line), and in response to the array (mean of $300-500$ ms after array onset, solid purple line). Polar grid lines indicate normalized minimum and maximum enhancement. Adjacent electrodes are shown from areas IPSO (B, C), V3B (D), and V3d (E). Note the reversal in field location from bottom to top quadrant in adjacent electrodes, indicating the high spatial specificity of HFB responses.

was considered to have a spatially-tuned IFP RF, if its tuning curve met three criteria. First, it had a significant task-evoked response at $\mathrm{RF}_{\mathrm{C}}$. Second, we determined whether the IFP responses were spatially selective by comparing the peak of the tuning curve (defined as $\mathrm{RF}_{\mathrm{C}}$ ) to the opposite location $\left(\mathrm{RF}_{\text {null }}\right)$ using a bootstrapped randomization. We generated a null distribution of randomized differences between $\mathrm{RF}_{\mathrm{C}}$ and $\mathrm{RF}_{\text {null }}$ means by drawing with replacement from a pool of all $\mathrm{RF}_{\mathrm{C}}$ and $\mathrm{RF}_{\text {null }}$ trials, including the number of $\mathrm{RF}_{\mathrm{C}}$ trials in one mean and the number of $\mathrm{RF}_{\text {null }}$ trials in the other. The difference between these randomly generated means was added to a null distribution of randomized differences. The quantile of the real difference $\left(\mathrm{RF}_{\mathrm{C}}-\mathrm{RF}_{\text {null }}\right)$ in the null distribution of randomized differences was taken as the $p$ value of the real difference. We rejected the null hypothesis that activity in $\mathrm{RF}_{\mathrm{C}}$ and $\mathrm{RF}_{\text {null }}$ trials were recorded from the same distribution of responses for $p$ values $<0.01$. And third, we determined whether each tuning curve was well described by a Gaussian function, where the variance explained by the fit of a Gaussian function was $>60 \%\left(r^{2}>0.6\right)$. Because task-evoked responses were recorded at locations arranged around a circular array at a constant eccentricity of $7^{\circ}$, the measured widths were converted from degrees of visual angle (dva) to circular distance around the arc: wid $=\mathrm{dva} \times$ $2 \pi 7^{\circ} / 360$. A few sites were excluded due to exceptionally wide variance of the Gaussian fit (excluded if $\sigma>240 \mathrm{dva} N=6$ ). Across all sites that met these criteria, the median $\sigma$ was $52 \mathrm{dva}$, which corresponds to an arc length of $6^{\circ}\left(\min =2^{\circ}, \max =17^{\circ}\right)$. Spatial tuning was similarly determined for delay and late array activity by comparing HFB responses when attention was directed to $\mathrm{RF}_{\mathrm{C}}$ (or neighboring locations) compared with $\mathrm{RF}_{\text {null. }}$ We refer to the spatial tuning properties during the delay as "memory field", and those in response to the attended (vs unattended) array as "attention field" (for examples, see Fig. 3).

Spatial tuning functions were generated by centering the mean power at each location on $\mathrm{RF}_{\mathrm{C}}$, and the tuning width was measured as half the area under the normalized tuning curve. Since subjects had different numbers of cue locations, we found the cubic spline interpolation of each tuning curve using the least common multiple of the subjects' location counts, which allowed us to compare spatial tuning of HFB responses from all recording sites within a cortical area. Each tuning curve was then normalized to its peak. The population response is shown as the mean of the smoothed, normalized tuning curves within each area. Error bars correspond to the $95 \%$ CIs of bootstrapped distributions generated by resampling 500 times with replacement from trials in each condition at each site.

Response onset latencies. For each electrode, the onset latency of HFB responses was measured as the time-to-half-peak at $\mathrm{RF}_{\mathrm{C}}$ in response to the cue, following analytical steps as in Lee et al. (2007). We first smoothed the HFB time series of each trial at $\mathrm{RF}_{\mathrm{C}}$ with an $8 \mathrm{~ms} \sigma$ Gaussian kernel. A distribution of baseline trialwise means (blm) was generated by randomly selecting power values 1000 times from all the baseline times and trials, equivalent to the number of trials ( $N$ tr) and times $(\mathrm{Nti})$ at $\mathrm{RF}_{\mathrm{C}}$, then taking the mean over the Ntr to generate a distribution of 1000 randomized baseline time series. The response peak was defined as the maximum at $\mathrm{RF}_{\mathrm{C}}$ in the 50-250 ms following cue onset that was $>99.9 \%$ of the blm distribution $(p<0.001)$. To ensure that we measured elevated, increasing responses, we set the minimum response time $\left(\mathrm{L}_{0}\right)$ as the first time at least $50 \mathrm{~ms}$ after cue onset that the response was more 
than half the peak value. The response onset latency was then taken as the first time point between $\mathrm{L}_{0}$ and $250 \mathrm{~ms}$ after cue onset that the power exceeded half the peak. Only sites with response onset latencies during this time period were considered to have cue-evoked responses. To compare array onset latencies to cue responses, we also performed this analysis using array-evoked activity in the attend-to- $\mathrm{RF}_{\text {null }}$ condition, defined below.

Attentional modulation: magnitude and topography of effects. To determine the strengths of attentional modulation during the delay and in response to the array, we compared mean HFB power from trials when attention was directed to $R F_{C}$ (the attend-to- $\mathrm{RF}_{\mathrm{C}}$ condition) to trials when attention was directed away from $\mathrm{RF}_{\mathrm{C}}$ toward the opposite field location (the attend-to- $\mathrm{RF}_{\text {null }}$ condition). We compared these trial-wise means by calculating an attentional modulation index (MI) of the normalized means in each epoch. For each site, the time series of the responses in the attend-to- $\mathrm{RF}_{\mathrm{C}}$ and the attend-to- $\mathrm{RF}_{\text {null }}$ conditions were normalized to the maximum value in the $500 \mathrm{~ms}$ window following cue onset (for delay effects) or array onset. The population time series for each area was the mean of these normalized time series across sites. The modulation index was the mean difference between the normalized attend-to- $\mathrm{RF}_{\mathrm{C}}$ and the attend-to- $\mathrm{RF}_{\text {null }}$ time series in the time window of interest, yielding the proportion of the maximum response. A distribution of bootstrapped MI values was found for each area by repeating the MI calculation 1000 times after resampling with replacement from trials in the attend-to- $\mathrm{RF}_{\mathrm{C}}$ and attend-to- $\mathrm{RF}_{\text {null }}$ conditions.

$\mathrm{MI}$ values were determined for each site, and sites were assigned to an enhanced $(\mathrm{MI}>0)$ or suppressed $(\mathrm{MI}<0)$ group within each area, and then averaged across sites to yield population data. Note that the assignment of sites to these groups did not rely on a significance test, and was presumed to include noise around zero.

The MI values during the delay and in the late array window were mapped onto brain surfaces and combined across subjects onto a surface in common space to yield their topography. Specifically, electrode coordinates of each subject were first identified in their native brain space then realigned to a normalized brain. For sites with a response field, the topography of attentional modulation effects during the delay and late array windows across subjects were plotted in this common space with color indicating MI spread cortically using a Gaussian kernel of $4 \mathrm{~cm}$. Large dots denote the topographic sites, and small dots the nontopographic ones.

Attentional modulation: latencies. Attentional modulation latencies were calculated based on the time courses of HFB responses evoked by the array in the attend-to- $\mathrm{RF}_{\mathrm{C}}$ condition versus the attend-to- $\mathrm{RF}_{\text {null }}$ condition. Time series were averaged across recording sites from the same area with an enhanced (or separately for suppressed) modulation index to yield population data; the modulation latencies were determined based on these population data. The modulation latency was defined as the first time point in a series of at least 50 consecutive milliseconds after the array onset latency (defined above as the time to half peak of the response at $\mathrm{RF}_{\text {null }}$ ) during which the responses in the attend-to- $\mathrm{RF}_{\mathrm{C}}$ condition were greater (or smaller in the case of suppressive effects) than in the attend-to- $\mathrm{RF}_{\text {null }}$ condition using the cluster method described in the following section. Our approach is similar to other studies measuring attentional modulation latencies, using the first of several consecutive significant time points (Gregoriou et al., 2009; Buffalo et al., 2010); however, we required longer clusters of significance (50 ms compared with $30 \mathrm{~ms}$ ) and smaller time bins ( $1 \mathrm{~ms}$ compared with $10 \mathrm{~ms}$ ) due to the differences in signal quality in HFB power compared with spiking activity.

Tests of statistical significance. To compare effects between areas, we generated bootstrapped distributions of the population means across sites within each area by randomly resampling 500 times with replacement from the trials in each condition. For example, for tuning widths we resampled from trials at each cue location to generate a randomized mean for each site at that cue location, then took the mean across the sites in the area, repeated 500 times to generate a distribution across the population of sites in that area. Using these distributions, we compared the means between every area using ANOVA, and the significance of each difference was determined by applying the Holm-Bonferroni sequential correction for multiple comparisons on the resulting $p$ values. In this method, a single target $\alpha$ level is applied across the set of tests, yielding a single $p$ value for all tests. Across all comparisons, the $p$ values from the ANOVA were ranked from the smallest to the largest and compared with a ranked $\alpha$ level determined by the following:

$$
\alpha_{\text {rank }}=\frac{\text { Target Alpha Level }}{n-\text { rank }+1},
$$

where $n$ was the number of tests, and the Target Alpha Level was set as 0.05 . For instance, 15 areas were included in the comparison of tuning widths (see Table 5), so the number of tests $n$ was $\left(\begin{array}{c}15 \\ 2\end{array}\right)=105$. In order of their rank, if a test had $p-v_{\text {alue }}$ rank $<\alpha_{\text {rank }}$, then that test was considered significant at the Target Alpha Level. The first test with $p-$ value $_{\text {rank }} \geq \alpha_{\text {rank }}$ was not significant, as well as all subsequent tests.

To determine whether an effect within an area was significantly different from zero, we found the $95 \%$ CI of the bootstrapped distribution. Areas with CI that did not overlap zero were significantly modulated $(p<0.05)$. We used Spearman's rank correlation to determine the relationship between cue-evoked tuning widths and latencies.

For measurements of sustained cue-evoked activity, we used a nonparametric cluster method (Maris and Oostenveld, 2007) to determine the number of sequential time points with significant enhancement relative to baseline. With this method, we set a threshold for significance $(p<0.05)$ and found clusters of sequential time points after the cue onset latency with significantly elevated power at $\mathrm{RF}_{\mathrm{C}}$. We used the quantile of the $\mathrm{RF}_{\mathrm{C}}$ power at each time point relative to a randomized distribution of baseline mean values as the test statistic at each time point. The cluster level statistic was the sum of the test statistics in the cluster. We compared veridical cluster level statistics to a null distribution of cluster level statistics generated by randomly assigning time points as eventrelated or baseline. Clusters of time points were significant if their veridical cluster level statistic was $>99 \%$ of the randomly generated cluster level statistics in the null distribution $(p<0.01)$.

To determine the attentional modulation latencies after array onset, we repeated the assessment of sustained activity but used the time series after the array onset and compared the attend-to- $\mathrm{RF}_{\mathrm{C}}$ condition to the attend-to- $\mathrm{RF}_{\text {null }}$ condition rather than to baseline. The latency of attentional modulation was the first time point of the first cluster after the array onset latency when attend-to- $\mathrm{RF}_{\mathrm{C}}$ was greater than attend-to$\mathrm{RF}_{\text {null }}$ (or smaller in the case of suppression effects).

Only areas with at least half of the bootstrapped calculations yielding a modulation latency were included in the groupwise comparison, thus areas ISP4+, frontal eye fields (FEF), and the nontopographic regions of occipital cortex were excluded from the group of modulation latencies. For area V1d/v enhanced sites, the distribution of bootstrapped modulation latencies was bimodal, so we separated the population of those latencies into two groups, which had an early (V1c1) and a late (V1c2) component. The distributions for $\mathrm{V} 1 \mathrm{cl}$ and $\mathrm{V} 1 \mathrm{c} 2$ were used in the groupwise comparisons.

\section{Results}

We recorded IFPs from 758 subdural electrodes implanted over parietal, occipital, temporal, and frontal cortex in 8 patients, who underwent presurgical epilepsy evaluation (Table 1; Fig. 1) while performing a spatial attention task. We eliminated 122 electrode channels that were compromised because of noise or epileptiform activity, yielding 636 channels for analysis.

\section{Electrode localization}

In each patient, structural MRI and CT images of the implanted electrodes were used to reconstruct their locations in occipital $(N=54)$, temporal $(N=170)$, parietal $(N=280)$, and frontal cortex $(N=132)$. To relate electrode positions more specifically to topographically organized areas of the visual system, we combined the structural MRI of each individual patient with a probabilistic atlas of visuospatial topographic cortex (Wang et al., 
2015). Electrode locations from all patients in relation to this probabilistic atlas are shown in Figure 1, rendered onto the left hemispheric surface of a standard brain and displaying posterior, lateral, and medial views. One hundred and thirty-three electrodes were located in the topographic visual system, including in early visual (V1-V3d/v, $N=36$ ), dorsal extrastriate (V3A/B, TO1-2, $N=24)$, ventral extrastriate (hV4, LO1-2, VO1-2, PHC1-2, $N=24$ ), and posterior parietal cortex, particularly in areas along the intraparietal sulcus (IPS; $N=42$ ), as well as in the superior parietal lobule (SPL1, $N=3$ ), and in frontal cortex (FEF, $N=4$ ). The remaining 503 electrodes were implanted outside visuospatial topographic areas. Using the Harvard-Oxford parcellation that differentiates cortical areas using anatomical markers (Desikan et al., 2006), these electrodes were broadly localized by lobe into occipital, temporal, parietal, and frontal categories. Because we did not find systematic differences in our analyses within a given category, results were combined by lobe (designated "Nontopographic, occipital" etc.). The electrodes in nontopographic cortex were distributed across parietal $(N=235)$, temporal $(N=134)$, and frontal lobes $(N=128)$, with only six electrodes in the occipital lobe located outside topographic cortex.

\section{Task design and behavioral results}

The patients were tested in a variant of the Eriksen flanker task (Eriksen and Eriksen, 1974; Eriksen, 1995), a classical spatial attention task that we also use in parallel monkey electrophysiology studies (Saalmann et al., 2012). Each trial of the task (Fig. 2A) was initiated by an auditory tone and the presentation of a fixation point on a computer monitor. After a fixation period of $1100 \mathrm{~ms}$, a cue was flashed briefly in a pseudo-randomly selected location arranged in a circular manner around the fixation point at a fixed eccentricity of $7^{\circ}$. The cue indicated with $100 \%$ validity the location of a subsequently presented target shape. After a variable delay period (300-700 ms), a circular array of barrel and bow tie shapes was presented, and the patients indicated with a left or right mouse button press which shape (i.e., barrel or bow tie) appeared at the cued location. Patients performed between 150 and 300 trials of this task (Table 1) and achieved high accuracies ranging from 83 to $96 \%$ (mean $=93 \pm 2 \%$ ). Importantly, the patients showed the classical flanker effect, with higher accuracies for targets that were flanked by congruent shapes than targets that were flanked by incongruent shapes (congruent: mean $=96 \pm$ $2 \%$, incongruent: mean $=90 \pm 3 \%$; $t$ test, $p<0.04$ ). This behavioral pattern indicates that the patients were engaged in the task and able to successfully perform it. To characterize the temporal dynamics of visual processing and its influences by attentional task demands, we report here on electrophysiological results from three epochs of the flanker task: cue-evoked (i.e., "bottomup" visual stimulation), delay period-related (i.e., maintenance of location information in the absence of visual stimulation), and array-evoked (i.e., the selection of behaviorally relevant stimuli among distracters).

\section{Spatial selectivity of cue-evoked HFB RFs}

We first examined the spatial selectivity of event-related power fluctuations of the IFPs recorded from each electrode. A representative example of a response profile from an IFP evoked by cue stimuli is shown in Figure 2. The recording site was located in left dorsal V3 (cortical location shown in Fig. $3 A$, electrode E). Cueevoked power modulations (50-250 ms after the cue onset) were compared with a baseline period (200 ms before cue onset). Averaged across all trials, a cue-evoked enhancement in power was observed across a broad band of high frequencies $(30-200 \mathrm{~Hz})$ with a concomitant suppression of power in a narrow band of lower frequencies (7-20 Hz; Fig. 2B), similar to typical profiles of IFP power fluctuations in response to visual stimuli previously reported in ECoG studies (Lachaux et al., 2005).

By examining power modulations relative to baseline as a function of time, we found that cue and array stimuli evoked a robust increase in the HFB power with a precise temporal profile marking the onset of the visual stimulation (Figs. $2 C, D$, top, $3 E$ ). In this report, we focus our analyses on modulations in HFB power between 70 and $200 \mathrm{~Hz}$ to exclude frequency bands that have been shown to have oscillatory properties such as gamma, beta, $\alpha$ or theta activity (Fries, 2009; Engel and Fries, 2010; Lisman and Jensen, 2013). However, control analyses on broadband activity that included gamma and beta frequency bands with the HFB responses yielded similar results. For the example electrode from dorsal V3, we sorted HFB responses in each trial based on cue location and found that the highest power was consistently evoked by the cue presented in positions 5 and 6 in the lower right quadrant (Figs. 2D, center, 3E, orange polar plot). Cues presented at locations further from the peak locations exerted continuously smaller HFB responses, thereby showing the typical profile of the cross section of a response field, which presents as a spatial tuning curve (Fig. $2 D$, right). Thus, the visually-evoked increases in HFB power recorded from this site were highly spatially specific, constituting a contralateral ECoG HFB response field. We defined the location that evoked the strongest $\mathrm{HFB}$ responses as the $\mathrm{RF}_{\mathrm{C}}$ (Fig. 3E, position 6) and the opposite field location as $\mathrm{RF}_{\text {null }}$ (Fig. $3 E$, position 13$)$. It is noteworthy that trialwise responses for each cue position were reliable, with consistently stronger responses at $\mathrm{RF}_{\mathrm{C}}(412 \pm 32 \%$ of baseline, bootstrap randomization test $p<$ 0.001 ) and consistently weaker or absent responses at the opposite field location $\left(\mathrm{RF}_{\text {null }}, 0.4 \pm 3 \%\right.$ of baseline, $\left.p=0.8\right)$.

Cue-evoked HFB responses showed a high degree of spatial specificity across cortex, both within topographic visual cortex and outside of topographic areas. We obtained distinct spatial profiles even from adjacent electrodes, as illustrated in Figure 3 for electrodes that were part of a strip with $10 \mathrm{~mm}$ spacing. In addition to the example V3d electrode (Fig. 3A, electrode E), three nearby electrodes with ECoG HFB response fields were implanted in areas IPSO (Fig. $3 A$, electrodes B and C, separated by $10 \mathrm{~mm}$ ), and in V3B (Fig. $3 A$, electrode D bordering V3A, separated from $\mathrm{C}$ and $\mathrm{E}$ by 10 and $14 \mathrm{~mm}$ respectively). We did not find HFB response fields in two other electrodes of this strip (Fig. $3 A$, blank circles). The peaks of the HFB response fields shifted from position 5, just below the right horizontal meridian (Fig. $3 B$ ) to position 3 in the top right quadrant (Fig. $3 C$ ) within IPS0, and from position 3 in the top right quadrant within V3B (Fig. $3 D$ ) to position 6 in the bottom right quadrant of V3d (Fig. $3 E$ ). This topographic pattern of peak responses reflects the visual field sign reversals of the underlying topographic maps (Konen and Kastner, 2008; Silver and Kastner, 2009; Arcaro et al., 2011; Wang et al., 2015). Thus, HFB responses reflected activity from spatially selective, local neuronal populations, and these signals did not appear to be compromised by volume conduction from more distant sites (Buzsáki et al., 2012), corroborating and extending previous reports on the specificity of HFB responses (Crone et al., 1998; Canolty et al., 2007; Parvizi et al., 2012). The spatial selectivity of HFB responses across the human visual system formed the basis for our quantitative analyses of the temporal dynamics and modulatory effects of selective attention on baseline and visually-evoked activity. 
A

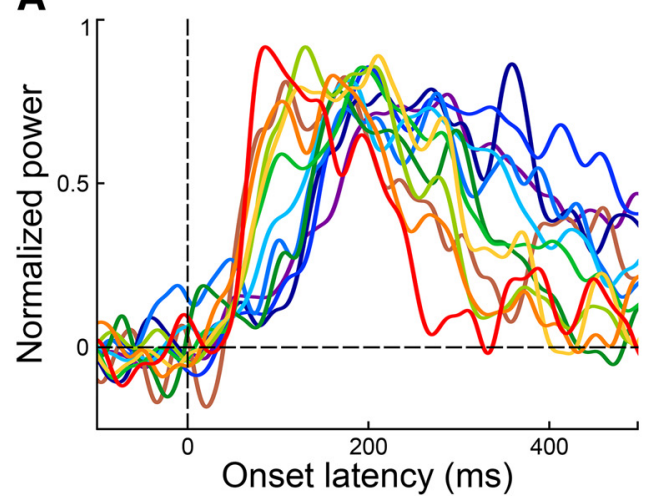

B

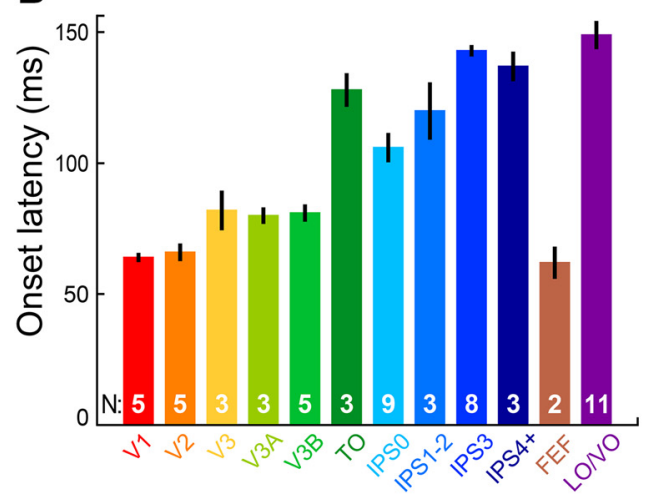

C

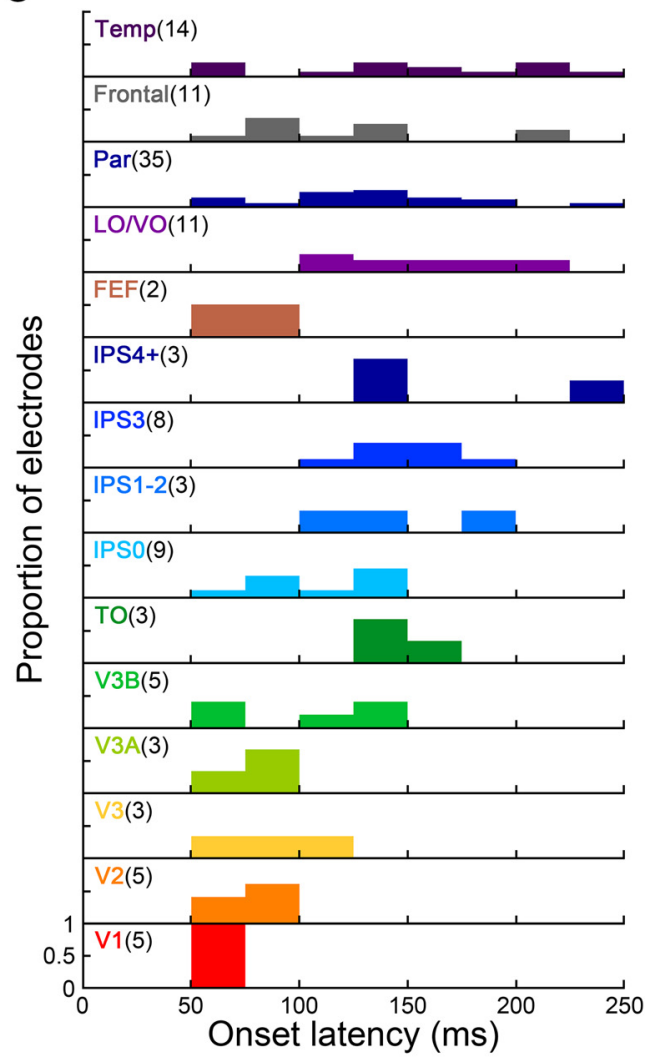

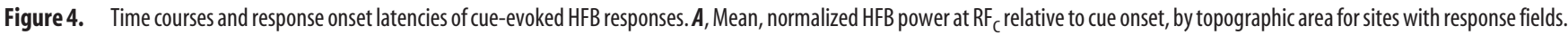
Color-codes for areas are indicated in $\boldsymbol{B}$. B. Mean response onset latency $\pm 95 \% \mathrm{Cl}$ by area. $N$ indicates the number of sites per area included in the analysis. $\boldsymbol{C}$, Response onset latencies of sites with response fields by area. Number of sites per area is indicated in parentheses.

Next, we determined the spatial tuning properties of cueevoked HFB responses based on the following criteria. First, for each recording site, we required responses to be visually selective such that cue-evoked HFB power increased significantly relative to baseline in response to at least one cue presentation location, as well as significant differences between cue-evoked responses at the preferred location $\left(\mathrm{RF}_{\mathrm{C}}\right)$ compared with the opposite location $\left(\mathrm{RF}_{\text {null }}\right)$. Second, we required that the response profile of the spatial tuning curve centered on $\mathrm{RF}_{\mathrm{C}}$ had a regular shape (i.e., a Gaussian fit centered on $\mathrm{RF}_{\mathrm{C}}$ explained at least $60 \%$ of the variance, and the tuning widths were $<240^{\circ}$ of visual angle). And third, to capture cue-evoked spatial tuning only (and not delayrelated tuning), we determined whether the response onset latency at $\mathrm{RF}_{\mathrm{C}}$ was within $50-250 \mathrm{~ms}$ of cue onset (latencies are discussed in the following section).

Using these criteria, $45 \%$ of electrodes located in topographic areas exhibited spatially-tuned, cue-evoked responses (60/133) with a well defined response field. The vast majority of these had their $\mathrm{RF}_{\mathrm{C}}$ in the contralateral hemifield $(58 / 60,97 \%)$. Additionally, in ventral and dorsal parts of visual areas V1-V3, spatial tuning was predominantly limited to the respective upper and lower visual field quadrants. Eighty-two percent, or 9/11 of the dorsal sites had their $\mathrm{RF}_{\mathrm{C}}$ in the lower contralateral quadrant, and $2 / 2$ of the ventral sites had their $\mathrm{RF}_{\mathrm{C}}$ in the upper contralateral quadrant. Of the recording sites outside of topographic visual areas, $12 \%$ exhibited spatially tuned, cue-evoked HFB responses (60/503), typically with their $\mathrm{RF}_{\mathrm{C}}$ contralateral to the implanted hemisphere $(46 / 60,77 \%)$. These sites were located in parietal $(N=$ 35 selective, 27 with contralateral $\left.\mathrm{RF}_{\mathrm{C}}\right)$, temporal $(N=14$ selective,
12 with contralateral $\mathrm{RF}_{\mathrm{C}}$ ), and frontal lobes $(N=11$ selective, 7 with contralateral $\mathrm{RF}_{\mathrm{C}}$ ). Except if noted otherwise, only the sites with a cue-evoked RF were included in further analyses.

\section{Cue-evoked response onset latencies}

We then examined the temporal dynamics of feedforward processing across the human visual system by analyzing HFB cue response onset latencies at $\mathrm{RF}_{\mathrm{C}}$ in topographic and nontopographic areas. We defined onset latency as the time to half peak of the power increase at $\mathrm{RF}_{\mathrm{C}}$ in response to the cue (Lee et al., 2007). For each recording site, we compared the mean time series of $\mathrm{HFB}$ power at $\mathrm{RF}_{\mathrm{C}}$ to a bootstrapped distribution of baseline means, finding the peak power in the cue interval that was greater than at least $99.9 \%$ of the bootstrapped baseline distribution. The onset latency was taken as the first time point at which the power was greater than half the peak. In the example area V3d electrode, the cue-evoked responses at $\mathrm{RF}_{\mathrm{C}}$ were highly consistent across trials and had a reliable onset latency of $59 \pm 8 \mathrm{~ms}$ (Fig. $2 D$, top). As expected from monkey single-unit recording studies (Schmolesky et al., 1998), HFB latencies increased systematically across the ventral and dorsal processing pathways (Fig. 4; Tables 2, 3).

Response onset latencies increased along the dorsal pathway from early visual areas (V1-V3d/v mean $=73 \pm 4 \mathrm{~ms})$ to dorsal extrastriate areas $(\mathrm{V} 3 \mathrm{~A} / \mathrm{B}$ and TO1-2 mean $=107 \pm 9 \mathrm{~ms}, p<$ 0.05 ; Table 3 shows all area-wise comparisons) and IPS0 (106 \pm 5 $\mathrm{ms})$. IPS0 latencies were faster than those in more anterior IPS areas. Response onset latencies in the ventral pathway were quite long, with ventral extrastriate area responses $($ mean $=149 \pm 5$ 
Table 2. Response onset and attentional modulation latencies by area

\begin{tabular}{lcl}
\hline $\begin{array}{l}\text { Brain } \\
\text { areas }\end{array}$ & $\begin{array}{l}\text { Cue response } \\
\text { latency, } m s\end{array}$ & $\begin{array}{l}\text { Attention latency, enhanced, } \\
\text { ms (suppressed) }\end{array}$ \\
\hline V1d/v & $64 \pm 1$ & $83 \pm 9,315 \pm 33(68 \pm 7)$ \\
V2d/v & $66 \pm 3$ & $295 \pm 16$ \\
V3d/v & $82 \pm 7$ & $233 \pm 12$ \\
V3A & $80 \pm 2$ & $246 \pm 8$ \\
V3B & $81 \pm 2$ & $268 \pm 22$ \\
T01-2 & $128 \pm 6$ & $129 \pm 3$ \\
IPS0 & $106 \pm 5$ & $156 \pm 18$ \\
IPS1-2 & $120 \pm 10$ & $119 \pm 22$ \\
IPS3 & $143 \pm 1$ & $225 \pm 11(265 \pm 142)$ \\
IPS4-5, SPL1 & $137 \pm 5$ & - \\
FEF & $62 \pm 5$ & - \\
vExtrastriate & $149 \pm 5$ & $172 \pm 7$ \\
Parietal & $100 \pm 3$ & $124 \pm 7$ \\
Frontal & $84 \pm 5$ & - \\
Temporal & $109 \pm 5$ & $223 \pm 16$
\end{tabular}

Numbers in the second column refer to the response onset latencies for each area (mean $\pm 95 \%$ Cl). Numbers in the third column refer to the attentional modulation latencies with suppression effects in parentheses. - Denotes areas without significant attentional modulation.

ms) on the order of those in the anterior IPS, and slower than in dorsal extrastriate and posterior IPS. These findings were not only observed in the population data, but they were remarkably consistent across the four individual patients with extensive electrode coverage of the visual system (results not illustrated). Interestingly, as in previous monkey studies (Schmolesky et al., 1998), area FEF had a fast latency of $62 \pm 5 \mathrm{~ms}$, on the order of the population latencies in early visual cortex. This fast latency likely reflects projections from the superior colliculus that bypass the cortex. Although this latency was obtained from only two sites, these fast latencies were quite consistent (Fig. 4C), and they were recorded from two patients (S3 and S6). Conduction delays between subsequent processing stages along the dorsal pathway were estimated to be on the order of $\sim 15 \mathrm{~ms}$ by examining the progression from V1-V2-V3-V3A-IPS0 (Table 2). In nontopographic sites, response onset latencies in the frontal ( $84 \pm 5 \mathrm{~ms}$ ), parietal $(100 \pm 3 \mathrm{~ms})$, and temporal lobes $(109 \pm 5 \mathrm{~ms})$ were slower than early visual areas and faster than the anterior topographic IPS and ventral extrastriate areas (Fig. 4C).

To determine whether the cue onset latencies were biased by particular stimulus properties such as shape and size, we also compared array onset latencies of trials in which attention was not at $\mathrm{RF}_{\mathrm{C}}$ (the attend-to- $\mathrm{RF}_{\text {null }}$ condition) to the cue onset latencies, and found no differences in latencies across the topographic areas ( $t$ test, $p=0.6$ ). Thus, response onset latencies did not appear to depend on the different stimulus configurations used in our study.

Together, the temporal dynamics of cue-evoked responses along the dorsal and ventral visual pathways were consistent with the notion of a hierarchical feedforward architecture of visual processing.

\section{Attentional modulation effects and their topography}

To determine dynamic task-related modulations of visual processing and probe feedback effects, we examined attention effects on baseline activity in the absence of visual stimulation (i.e., during the delay) and in response to the array by comparing responses from trials when attention was allocated at $\mathrm{RF}_{\mathrm{C}}$ to trials when attention was allocated at $\mathrm{RF}_{\text {null }}$, similar to approaches typically taken in monkey physiology studies (Reynolds and Chelazzi, 2004). First, we characterized the different types of attentional modulation and their topography across the human visual system and nontopographic cortex. The vast majority of attention effects were enhancement of HFB responses during the delay and in response to the array, as shown for an example electrode located in area TO and for the TO population response in Figure 5 (top). Such enhancement effects were not only observed at $\mathrm{RF}_{\mathrm{C}}$, but typically had a spatial extent that was similar to the cue-evoked HFB RF, as can be seen in the examples shown in Figure 3 (modulation of array-evoked responses, solid purple plot; modulation of responses during delay, dashed purple plots). Collectively, the modulation at the different spatial locations relative to the response at $\mathrm{RF}_{\text {null }}$ gave rise to an attention field. Similarly, response enhancement during the delay was spatially tuned and gave rise to a memory field (see section on spatial tuning for further results).

Attention and memory fields were observed in many extrastriate sites but were markedly absent in early visual cortex (Fig. 6), especially during the delay. Of the sites in early visual cortex that had a RF, only one site showed significant attentional modulation during the delay $\left(N_{\text {delay }}=1 / 13,8 \%\right)$. Ventral extrastriate areas also had a low proportion of sites with a significant delay enhancement effect $\left(N_{\text {delay }}=2 / 11,18 \%\right)$. In comparison, in dorsal extrastriate and IPS areas $\sim 50 \%$ of sites showed significantly enhanced delay activity (dorsal extrastriate: $N_{\text {delay }}=5 / 11,45 \%$; IPS0-2: $N_{\text {delay }}=6 / 12,50 \%$; IPS3-5 and SPL1: $N_{\text {delay }}=6 / 11$, $54 \%)$. Among nontopographic areas, $20 \%$ of the sites that showed cue-evoked spatial tuning exhibited significant modulation of activity during the delay $\left(N_{\text {delay }}=12 / 60\right)$. Early visual areas also had relatively few sites with a significant effect of attention in response to the array $\left(N_{\text {array }}=5 / 13,38 \%\right.$ in the late array period) compared with dorsal extrastriate areas and posterior IPS, which had a high proportion with a significant attentional enhancement during the late array period (V3A, V3B, TO1-2: $N_{\text {array }}=7 / 11,64 \%$; IPS0-2: $N_{\text {array }}=7 / 12,58 \%$ ).

It is notable that the topography of attentional enhancement effects during the delay and in response to the array was not identical (Fig. 6, red areas). In particular, although ventral extrastriate areas LO/VO had a low proportion of sites that showed significant enhancement during the delay (18\%), these areas had a majority of sites showing an enhancement effect in response to the array $\left(N_{\text {array }}=7 / 11,64 \%\right)$. In nontopographic parietal areas, only $15 \%$ of sites showed enhanced delay activity $(N=9 / 60)$, whereas $40 \%$ exhibited attentional enhancement in response to the array $(N=24 / 60)$. Conversely, although anterior IPS areas IPS4 + had a high proportion of sites with a significant effect during the delay (54\%), it had only a few sites with significant enhancement in response to the array $\left(N_{\text {array }}=3 / 11,27 \%\right)$. Thus, only dorsal extrastriate areas and posterior IPS had a majority of sites enhanced by attention during both the delay and in response to the array (dorsal extrastriate: delay $45 \%$, array 64\%; IPS0-2: delay $50 \%$, array $58 \%$ ).

We also observed attentional suppression effects during the delay or in response to the array, albeit less frequently (Fig. 6, green areas). The example electrode shown in Figure 5 (middle, left) was located in V1 and showed a reduction of $\sim 50 \%$ in HFB responses to the array when attention was directed to $\mathrm{RF}_{\mathrm{C}}$ compared with $\mathrm{RF}_{\text {null }}$. Attentional suppression has been previously observed in monkey physiology studies as a decrease of LFP power and spike-field coherence in gamma frequency bands (40-60 Hz; Chalk et al., 2010). Given that we used an array of stimuli it is likely that inhibitory center-surround interactions and top-down influences contributed to these effects (Ito and Gilbert, 1999; Angelucci et al., 2002; Bair et al., 2003; Ozeki et al., 2009; Zhang et al., 2014; Cox et al., 2017). A similar result was 
Table 3. Response onset latencies and attentional modulation latencies: significance by area

\begin{tabular}{|c|c|c|c|c|c|c|c|c|c|c|c|c|c|c|c|c|c|}
\hline \multirow[b]{2}{*}{ Response onset latencies } & \multicolumn{17}{|c|}{ Attentional modulation latencies } \\
\hline & $\mathrm{V} 1 \mathrm{~d} / \mathrm{v}$ & V1c1 & V1c2 & $\mathrm{V} 2 \mathrm{~d} / \mathrm{v}$ & $\mathrm{V} 3 \mathrm{~d} / \mathrm{v}$ & V3A & V3B & TO & IPSO & IPS1-2 & IPS3 & IPS4+ & FEF & LO/VO & Parietal & Frontal & Temporal \\
\hline \multicolumn{18}{|l|}{$\mathrm{V} 1 \mathrm{~d} / \mathrm{v}$} \\
\hline V1c1 & & & - & - & $\cdot$ & • & - & - & $\cdot$ & & - & & & - & $\cdot$ & & - \\
\hline V1c2 & & & & & * & * & * & * & * & * & * & & & * & * & & * \\
\hline $\mathrm{V} 2 \mathrm{~d} / \mathrm{v}$ & & & & & & & & * & * & * & & & & * & * & & $*$ \\
\hline $\mathrm{V} 3 \mathrm{~d} / \mathrm{v}$ & + & & & + & & & & * & * & * & & & & * & * & & \\
\hline V3A & + & & & + & & & & * & * & * & & & & * & * & & \\
\hline V3B & + & & & + & & & & * & * & * & & & & * & * & & * \\
\hline T0 & + & & & + & + & + & + & & & & $\cdot$ & & & & & & \\
\hline IPSO & + & & & + & + & + & + & - & & * & $\cdot$ & & & & & & • \\
\hline IPS1-2 & + & & & + & + & + & + & & + & & • & & & & & & • \\
\hline IPS3 & + & & & + & + & + & + & + & + & & & & & $*$ & * & & \\
\hline IPS4+ & + & & & + & + & + & + & + & + & + & & & & & & & \\
\hline FEF & & & & & - & - & - & - & - & - & - & - & & & & & \\
\hline LO/VO & + & & & + & + & + & + & + & + & + & & & + & & & & \\
\hline Parietal & & & & + & & & & & & - & - & - & + & - & & & \\
\hline Frontal & & & & + & & & & - & & - & - & - & + & - & & & \\
\hline Temporal & + & & & + & + & & & & & - & - & - & + & - & + & + & \\
\hline
\end{tabular}

Response onset latencies (lower triangle): significance after the Holm-Bonferroni sequential correction for multiple comparisons at target alpha level $p<0.05$. + Indicates that the latency of the column area was faster than the row area, and vice versa for - . Attentional modulation latencies (upper triangle): Significance after Holm-Bonferroni sequential correction for multiple comparisons at target alpha level $p<0.05$. ${ }^{*}$ Indicates that the latency of the column area was faster than the row area, and vice versa for $\cdot$. Blank cells indicate no significant difference between areas.

\section{Example Sites}
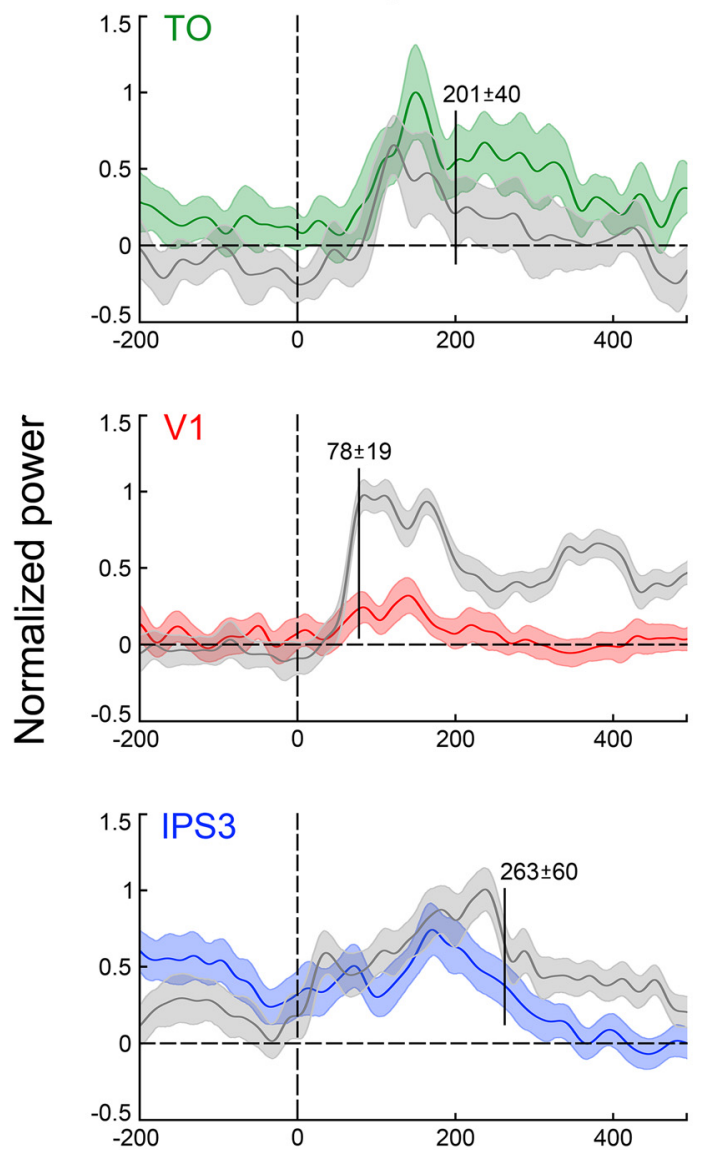

Population
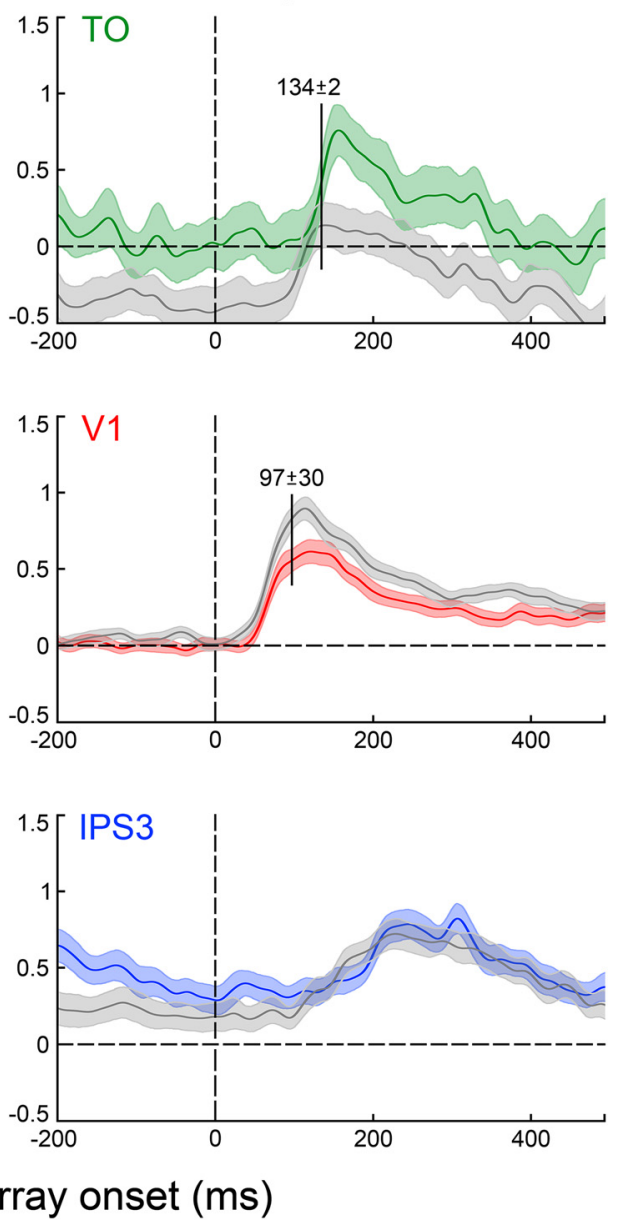

Figure 5. Examples of attentional modulation effects during the delay and in response to the array. Top, Attentional enhancement during the delay and in response to the array. Mean responses $\pm 95 \%$ Cl evoked by the array during attend-to- $\mathrm{RF}_{\mathrm{C}}$ (green) and attend-to- $\mathrm{RF}_{\text {null }}$ (gray) trials from an example T0 electrode (left) and the T0 population (right). Middle, Attentional suppression in response to the array for an example V1v electrode (left) and the V1 population (right). Bottom, Mixed effects of attentional enhancement and suppression for an example IPS3 electrode showing attentional enhancement during the delay, but suppression in response to the array (left). In the IPS3 population response (right), only the elevated delay effect persists, whereas suppression and enhancement effects in individual electrodes cancel each other out to result in no modulatory net effect in response to the array. Vertical lines indicate attentional modulation latencies. 

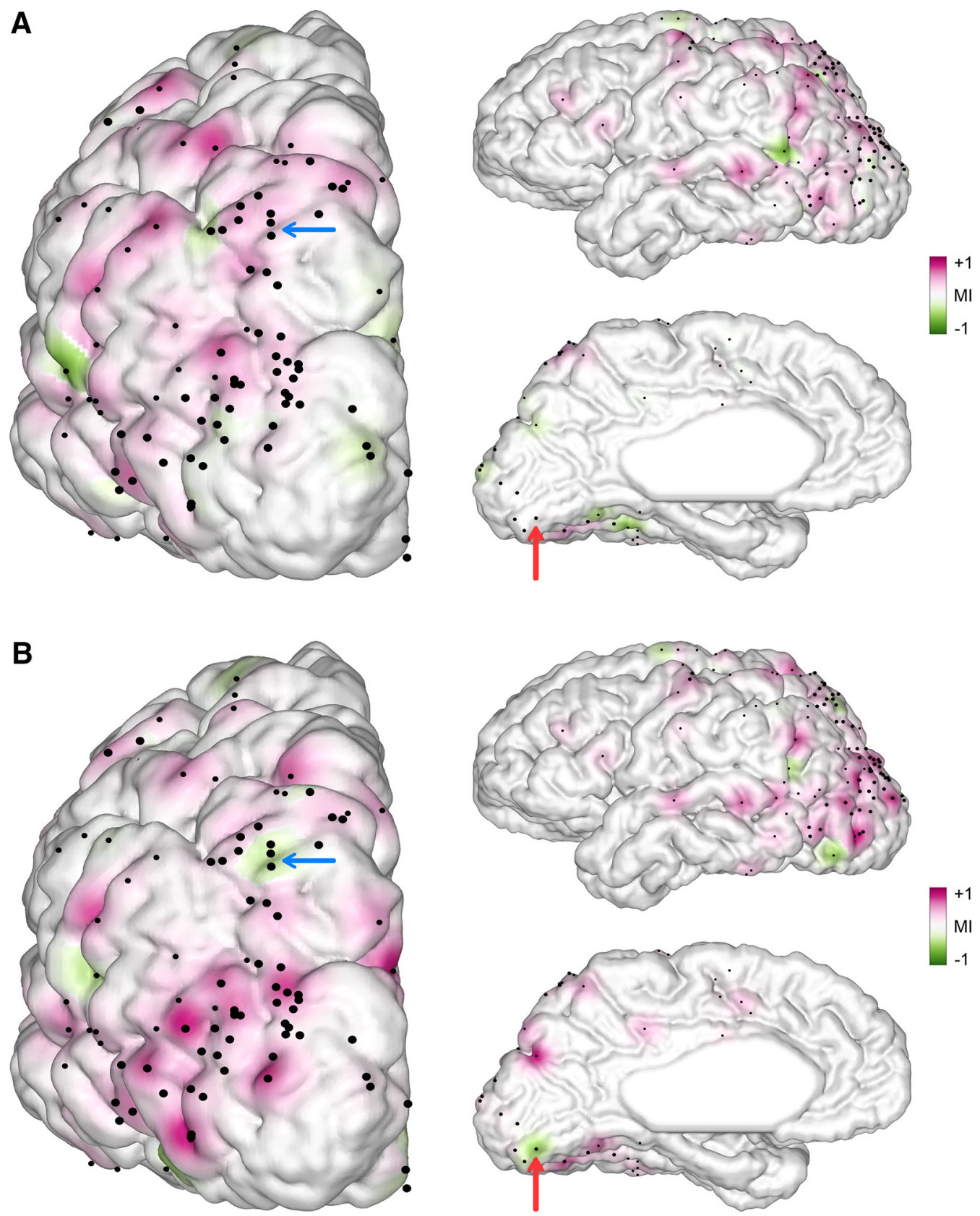

Figure 6. Topography of attentional modulation effects during the delay and in response to the array. Color indicates the Ml for sites with a response field across all subjects rendered onto a brain surface in standardized space, shown from posterior, lateral, and medial views. Large dots, Topographic sites; small dots, nontopographic sites. $\boldsymbol{A}$, Topography and strength of attentional modulation during the delay ( $200 \mathrm{~ms}$ before array onset, only trials with delays longer than $450 \mathrm{~ms}$ were included in the analysis). $\boldsymbol{B}$, Topography and strength of attentional modulation in response to the array in the late window ( $300-500 \mathrm{~ms}$ after array onset). Red areas, Enhancement effects; green areas, suppression effects.

obtained for the population of $\mathrm{V} 1 \mathrm{~d} / \mathrm{v}$ sites, with an overall suppression effect of $\sim 10 \%$ in response to the array (Fig. 5 , middle, right). Attentional suppression effects were also found in IPS areas (Fig. 6, green areas). Interestingly, array-related suppression in IPS could be observed with elevated delay activity, as shown in Figure 5 (bottom left) for an electrode located in area IPS3 (for sites with such effects, see Fig. 6A, B, blue arrows). Because both array-related attentional enhancement and suppression effects were found in this area, no net effect of modulation resulted in the population response (Fig. 5, bottom right; mean $=5 \pm 6 \%$ enhancement, bootstrap randomization test $p=$ $0.06)$.

\section{Strengths of attentional modulation effects}

Hierarchical top-down models assume modulatory attention effects to reverse the bottom-up processing hierarchy. One prediction of such a model is that effects of attention are stronger at advanced compared with early stages of visual processing. Therefore, we probed the strengths of modulatory effects across the human visual system as well as in nontopographic cortex. We quantified the attention effects obtained during the delay and in response to the array using a MI (defined as the difference between the mean power in attend-to- $\mathrm{RF}_{\mathrm{C}}$ and attend-to- $\mathrm{RF}_{\text {null }}$ conditions, normalized to the maximum response). The MI therefore calculates the modulation effect as the proportion of 

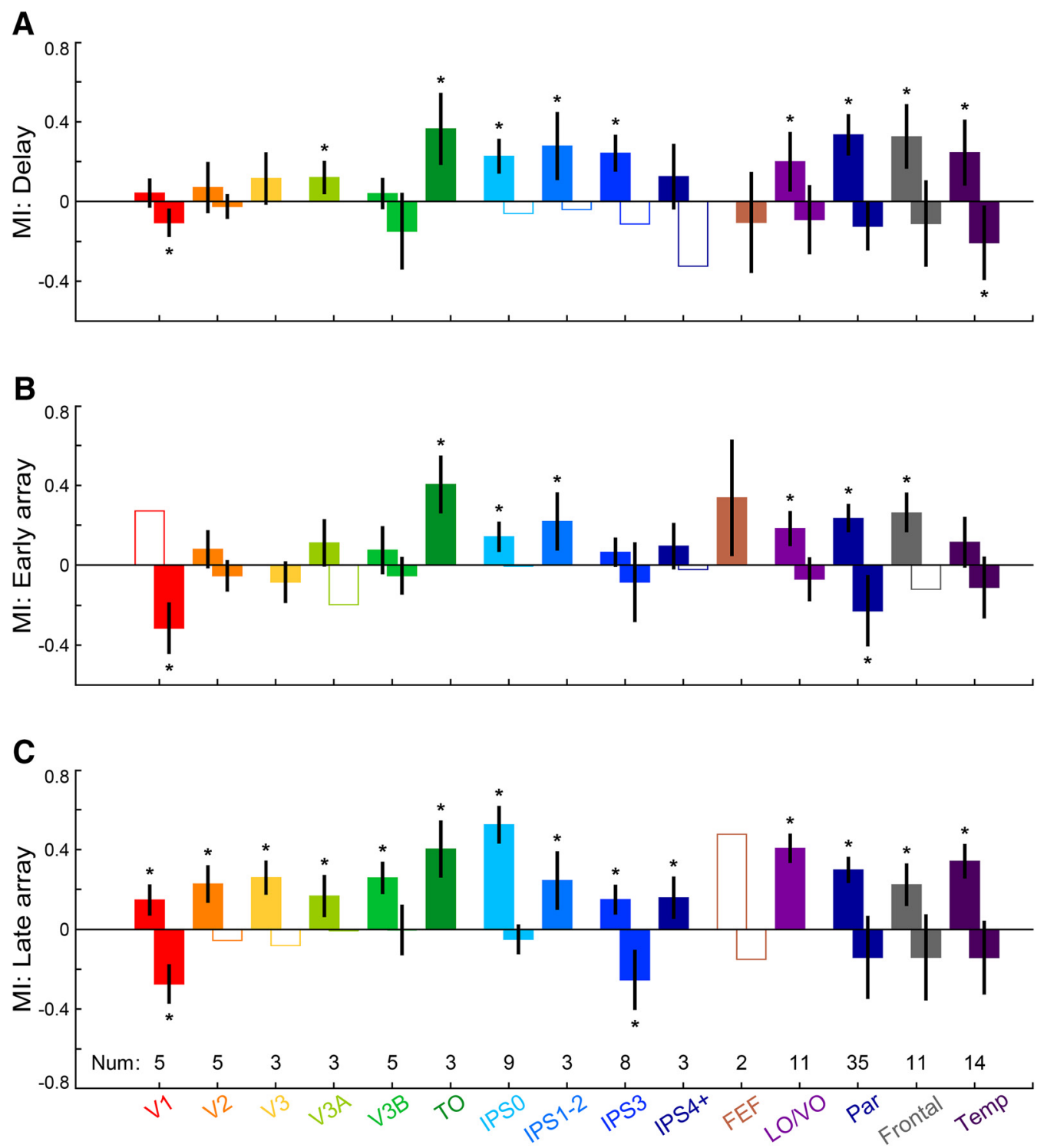

Figure 7. Strengths of attentional modulation during the delay and in response to the array. $A$, Attentional $\mathrm{Ml}$ in each area during the delay ( $200 \mathrm{~ms}$ before array onset) using trials with delays $>450 \mathrm{~ms}$. Mean modulation index $( \pm 95 \% \mathrm{Cl})$ shown separately for enhancement and suppression effects obtained in each area at the population level. *Indicates bootstrapped randomization test $p<0.05$ compared with 0. Outlined bars shown for areas with a single electrode exhibiting the effect. $\boldsymbol{B}$, As in $\boldsymbol{A}$ for modulation during the early array window ( $50-200 \mathrm{~ms}$ after array onset). $\boldsymbol{C}$, As in $\boldsymbol{A}$ for modulation during the late array window (300-500 ms after array onset).

the maximum HFB response. We calculated the MI for the delay period (200 ms before array onset, only including trials with cuetarget intervals $>450 \mathrm{~ms}$ to capture attention effects that were not contaminated by cue-evoked responses), early array (50-200 $\mathrm{ms})$, and late array period (300-500 ms). Positive values indicate enhancement effects (Fig. 6, red) and negative values indicate suppression effects (Fig. 6, green).

To compare the effects of attention between areas, we separately generated bootstrapped distributions of MIs using the population of sites with either enhanced or suppressed effects in each area. Importantly, sites were not assigned to those groups based on any measure of significance, but strictly based on whether their MI was positive or negative. Statistical analyses for each area were then performed on the population means of each of those groups. During the delay, we found significant enhancement effects of attention in dorsal and ventral extrastriate areas (V3A, TO1-2, LO1-2, hV4, VO1-2), as well as in IPS areas IPS0-3 (bootstrap randomization test, each $p<0.001$; Figs. $6 A$, red areas, $7 A$ ).
No significant enhancement effects were found in early visual areas $\mathrm{V} 1 \mathrm{~d} / \mathrm{v}, \mathrm{V} 2 \mathrm{~d} / \mathrm{v}$, or $\mathrm{V} 3 \mathrm{~d} / \mathrm{v}$, nor in dorsal extrastriate area $\mathrm{V} 3 \mathrm{~B}$ or anterior IPS areas IPS4-5, SPL1, and FEF (each $p \sim 0.1$; Figs. $6 A$, red areas, $7 A$ ). Of the areas with a significant effect, V3A $\left(\mathrm{MI}_{\text {delay }}=12 \pm 8 \%, N=3\right)$ and $\mathrm{LO}\left(\mathrm{MI}_{\text {delay }}=20 \pm 14 \%, N=8\right)$ showed weaker modulation during the delay than IPS areas (IPS0: $\mathrm{MI}_{\text {delay }}=23 \pm 8 \%, N=8$; IPS1-2: $\mathrm{MI}_{\text {delay }}=28 \pm 16 \%$, $N=2$; IPS3: $\mathrm{MI}_{\text {delay }}=24 \pm 8 \%, N=7$ ) and dorsal extrastriate area $\mathrm{TO}\left(\mathrm{MI}_{\text {delay }}=37 \pm 17 \%, N=3\right)$. The significance of each comparison is shown in Table 4.

Outside visual topographic cortex, we found significant population enhancement effects in parietal $\left(\mathrm{MI}_{\text {delay }}=34 \pm 10 \%\right.$, $N=23)$, frontal $\left(\mathrm{MI}_{\text {delay }}=33 \pm 16 \%, N=7\right)$, and temporal lobes $\left(\mathrm{MI}_{\text {delay }}=25 \pm 16 \%, N=8\right.$; each $p<0.001$; Figs. $6 A$, red areas, $7 A$ ), with modulatory effects similar in strength to higherorder topographic areas (Table 4).

Of the sites with a negative MI, attention significantly suppressed HFB power modulations in the population of $\mathrm{V} 1 \mathrm{~d} / \mathrm{v}$ 
Table 4. Attentional modulation during the delay and in response to the array: significance by area

\begin{tabular}{|c|c|c|c|c|c|c|c|c|c|c|c|c|c|c|}
\hline \multirow{2}{*}{$\begin{array}{l}\text { Attentional } \\
\text { modulation } \\
\text { strength, } \\
\text { delay }\end{array}$} & \multicolumn{14}{|c|}{ Attentional modulation strength, array } \\
\hline & $\mathrm{V} 1 \mathrm{~d} / \mathrm{v}$ & $\mathrm{V} 2 \mathrm{~d} / \mathrm{v}$ & $\mathrm{V} 3 \mathrm{~d} / \mathrm{v}$ & V3A & V3B & T0 & IPSO & IPS1-2 & IPS3 & IPS4+ & LO/VO & Parietal & Frontal & Temporal \\
\hline $\mathrm{V} 1 \mathrm{~d} / \mathrm{v}$ & & • & - & & - & - & - & & & & . & - & . & . \\
\hline $\mathrm{V} 2 \mathrm{~d} / \mathrm{v}$ & & & & * & & - & - & & * & * & - & - & & • \\
\hline $\mathrm{V} 3 \mathrm{~d} / \mathrm{v}$ & & + & & * & & $\cdot$ & $\cdot$ & & * & * & - & • & & • \\
\hline V3A & + & & & & - & $\cdot$ & $\cdot$ & $\cdot$ & & & $\cdot$ & $\cdot$ & $\cdot$ & $\cdot$ \\
\hline V3B & & & & - & & - & - & & * & * & - & - & * & • \\
\hline T0 & + & + & + & + & + & & - & * & * & * & & * & * & \\
\hline IPSO & + & + & + & + & + & & & * & * & * & * & * & * & * \\
\hline IPS1-2 & + & + & + & + & + & & & & & & - & & & \\
\hline IPS3 & + & + & + & + & + & & & & & & - & - & $\cdot$ & • \\
\hline IPS4+ & & & & & & - & - & - & - & & - & - & $\cdot$ & $\cdot$ \\
\hline LO/VO & + & + & + & & + & - & & - & & + & & * & * & * \\
\hline Parietal & + & + & + & + & + & & + & & + & + & + & & * & $\cdot$ \\
\hline Frontal & + & + & + & & + & & & & & + & + & & & $\cdot$ \\
\hline Temporal & + & + & + & + & + & & & & & + & + & & & \\
\hline
\end{tabular}

Delay period (lower triangle): significance after Holm-Bonferroni sequential correction for multiple comparisons at target alpha level $p<0.05$. + Indicates that the Ml of the column area was weaker than the row area, and vice versa for - . Array period (upper triangle): modulation index for attentional enhancement in the late array window (300-500 ms after array onset); significance after Holm-Bonferroni sequential correction for multiple comparisons at target alpha level $p<0.05$. * Indicates that the Ml of the column area was weaker than the row area, and vice versa for $\cdot$. Blank cells indicate no significant difference between areas. ${ }^{2}$

$\left(\mathrm{MI}_{\text {delay }}=11 \pm 7 \%, N=3\right), \mathrm{ISP} 4+\left(\mathrm{MI}_{\text {delay }}=33 \pm 27 \%, N=1\right)$, and nontopographic temporal lobe sites $\left(\mathrm{MI}_{\text {delay }}=21 \pm 18 \%\right.$, $N=6$; bootstrap randomization test, all $p<0.001$; Figs. $6 A$, green areas, $7 A$ ). Notably, although the positive effects were not always significant in these areas, when we examined the effect across all sites in each area we found no overall effect of attention during the delay $(\mathrm{V} 1 \mathrm{~d} / \mathrm{v}: p=0.06$, IPS4 $+: p=0.4$, nontopographic temporal sites: $p=0.8$ ).

Next, we investigated attentional modulation of array-evoked activity. Attention effects can typically be observed in later time windows, since the feedforward cascade of visual stimulation strongly activates sites within the visual system regardless of whether they are attended to or not. For the time period of $300-$ $500 \mathrm{~ms}$ after array onset, we found significant positive modulation effects in early, dorsal and ventral extrastriate visual areas $(p<0.001)$, as well as consistently strong effects in IPS areas $(p<$ 0.001 ; Fig. $7 C)$. The strength of the modulation generally increased across the cortical hierarchy through IPS0, with the weakest modulation in early visual areas (V1d/v MI $\mathrm{Mrray}=15 \pm 7 \%)$, and the strongest modulation in dorsal extrastriate area TO $\left(\mathrm{MI}_{\text {array }}=40 \pm 14 \%\right)$, ventral extrastriate areas $\mathrm{LO} / \mathrm{VO}\left(\mathrm{MI}_{\text {array }}\right.$ $=40 \pm 7 \%)$, and posterior parietal area ISP0 $\left(\mathrm{MI}_{\text {array }}=53 \pm 7 \%\right.$; Figs. $6 B$ red areas, $7 C$; significance of all comparisons shown in Table 4). Interestingly, the anterior IPS areas were as weakly modulated as early visual area $\mathrm{V} 1 \mathrm{~d} / \mathrm{v}$ (IPS4 $+\mathrm{MI}_{\text {array }}=16 \pm$ 10\%; Fig. 7C; Table 4). We also observed significant suppression in areas $\mathrm{V} 1 \mathrm{~d} / \mathrm{v}$ and IPS3 $\left(\mathrm{MI}_{\text {array }}=-27 \pm 9 \%\right.$ and $-25 \pm 14 \%$, respectively; Fig. $7 C$ ), which were the only areas with this effect either across the population or from individual sites (sites with significant array suppression in V1d/v: $N_{\text {array }}=2$ from patient $\mathrm{S} 1$; IPS3: $N_{\text {array }}=1$ from S5). In contrast, during the early array period, when attention effects and visual onset activity interact, only topographic areas TO, IPSO-2, and LO/VO were significantly modulated (bootstrapped mean $\pm 95 \% \mathrm{CI}$, TO $\mathrm{MI}_{\text {array }}=$ $41 \pm 14 \%$; IPS0 $\mathrm{MI}_{\text {array }}=14 \pm 7 \%$; IPS1-2 $\mathrm{MI}_{\text {array }}=22 \pm 14 \%$; LO/VO $\mathrm{MI}_{\text {array }}=18 \pm 8 \%$; Fig. $7 B$ ).

In summary, TO, IPSO -2 , and $\mathrm{LO} / \mathrm{VO}$ exhibited stronger attentional modulation effects than early visual and anterior IPS areas both during the delay and in the late array window, and these were the only topographic areas that were significantly modulated during their early response to the array. Although the stronger attention effects in extrastriate and posterior parietal cortex relative to early visual cortex are consistent with hierarchical top-down models of attention, the weak or absent attention effects in the anterior IPS and frontal cortex, particularly during visual processing, are in conflict with such models.

\section{Attentional modulation latencies}

Just as the temporal order of visual onset responses informs about the temporal dynamics of feedforward visual processing, the timing of selective processing after the array onset provides insight into the temporal dynamics of feedback attentional modulation. Hierarchical top-down models predict that the latencies of attentional modulation systematically increase from advanced to early processing stages as a further indication for a reversal of the processing hierarchy during attentional selection. To determine the latency of attentional modulation after array onset, we examined the population time courses of each area sorted by modulation effects (i.e., enhancement or suppression based on each sites' MI in the late array window). First, we determined which time points showed a significant effect of attention in response to the array (attend-to- $\mathrm{RF}_{\mathrm{C}}>$ attend-to- $\mathrm{RF}_{\text {null }}$, bootstrap randomization $p<0.05)$. Then, we identified clusters of consecutive significant time points after array onset that lasted for at least $50 \mathrm{~ms}$ (Maris and Oostenveld, 2007). The first time point in the first cluster of significant ones after array onset was defined as attentional modulation latency (see Materials and Methods for more details). To compare latencies across areas, we generated bootstrapped distributions of attentional modulation latencies by resampling 500 times, with replacement, from trials in each condition by site and recalculating the latency based on that set of trials. We determined whether two areas had significantly different latencies by comparing the population means of the distributions, then applying Holm's sequential Bonferroni correction for multiple comparisons at $\alpha$ level $p<0.05$ across all the comparisons. The results are summarized in Tables 2 and 3.

Consistent with the idea that feedback signals are generated in higher-order cortex and modulate early sensory processing areas via corticocortical feedback, we found that modulation latencies were longest in early visual cortex (Figs. 8, 9; Tables 2, 3). Modulation latencies were slowest in V1d/v (late component, $315 \pm$ $33 \mathrm{~ms})$, followed by V2d/v (295 $\pm 16 \mathrm{~ms}), \mathrm{V} 3 \mathrm{~d} / \mathrm{v}(233 \pm 12 \mathrm{~ms})$, 

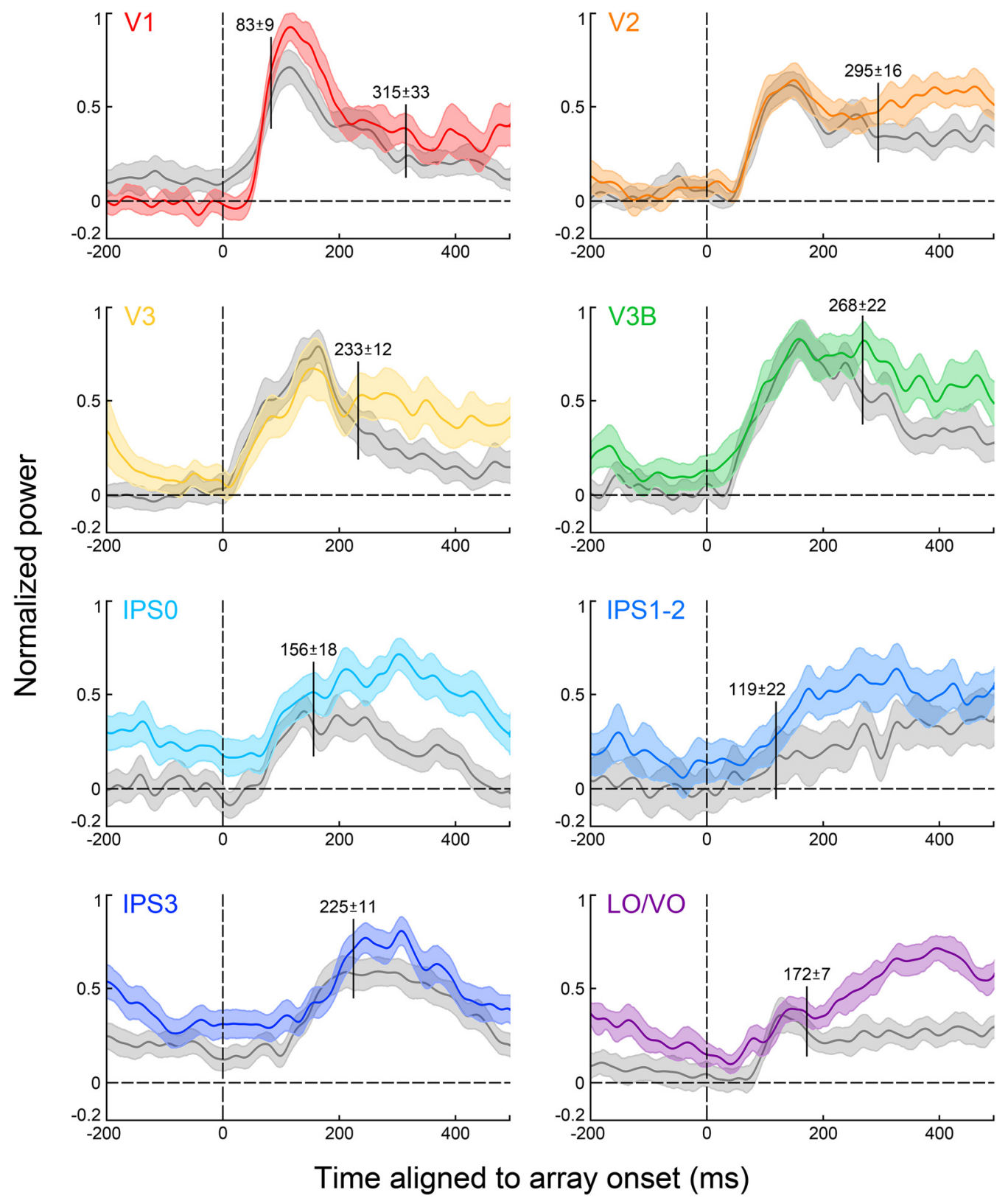

Figure 8. Time courses of array-evoked HFB responses. Mean, normalized power $( \pm 95 \% \mathrm{Cl})$ when attention was allocated at $\mathrm{RF}_{\mathrm{C}}$ (colored by area) and $\mathrm{RF}_{\text {null }}$ (gray), aligned to array onset. Topographic areas with significant response enhancement effects are shown (population responses). Vertical lines indicate attentional modulation latencies.

V3A $(246 \pm 8 \mathrm{~ms})$, and V3B $(268 \pm 22 \mathrm{~ms}$; significance of all comparisons shown in Table 3). The attentional modulation latencies in posterior IPS (IPS0: $156 \pm 18 \mathrm{~ms}$, IPS1-2: $119 \pm 22$ $\mathrm{ms})$, dorsal extrastriate area TO1-2 (129 $\pm 3 \mathrm{~ms})$ and ventral extrastriate areas $\mathrm{LO} / \mathrm{VO}(172 \pm 7 \mathrm{~ms})$ were significantly faster than those in early visual areas. However, the modulation latency in area IPS3, located anterior to IPS0-2, was significantly longer than the latencies in the posterior IPS and on the order of latencies in early visual areas (IPS3 latency $=225 \pm 11 \mathrm{~ms}$; Figs. 8, 9; Tables 2, 3). Although the more anterior IPS areas of IPS4+ had significant modulation effects (Fig. 7C), the responses were not robust across trials and sites, yielding $<50 \%$ of bootstrapped time series with a significant modulation effect. Therefore, the latencies calculated in this area were not considered significant (see Materials and Methods). However, it is worth mentioning that the trend of increasing latencies through the higher-order
IPS areas continued in IPS4+: of the bootstrapped time series where we were able to determine a modulation effect, the latency was even slower than IPS 3 and on the order of the slow V1d/v effects (latency $=352 \pm 22 \mathrm{~ms}$ from $41 \%$ of the bootstrapped time series). Further, modulation latencies could not be determined in FEF due to the absence of modulation effects (Fig. 7). Thus, the pattern of attentional modulation latencies did not strictly follow the concept of top-down feedback from higher to lower order cortex, with the fastest latencies found instead in intermediate areas of the processing streams.

In $\mathrm{V} 1 \mathrm{~d} / \mathrm{v}$, we found that the distribution of modulation latencies was bimodal, reflecting two components (Fig. 9B, red traces). A fast component indicated the effect of attention as early as 80 $\mathrm{ms}$ in V1 after array onset ( $83 \pm 9 \mathrm{~ms}$; Fig. $8, \mathrm{~V} 1)$, which was the fastest effect of attentional enhancement that we observed across all areas. Although these responses are too fast to reflect cortico- 
A

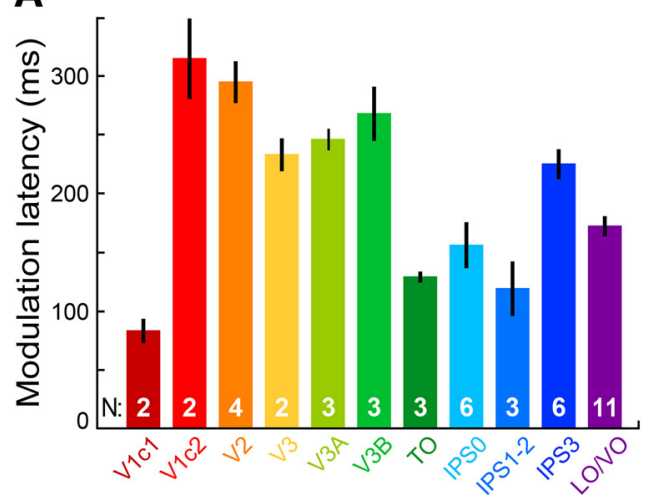

B

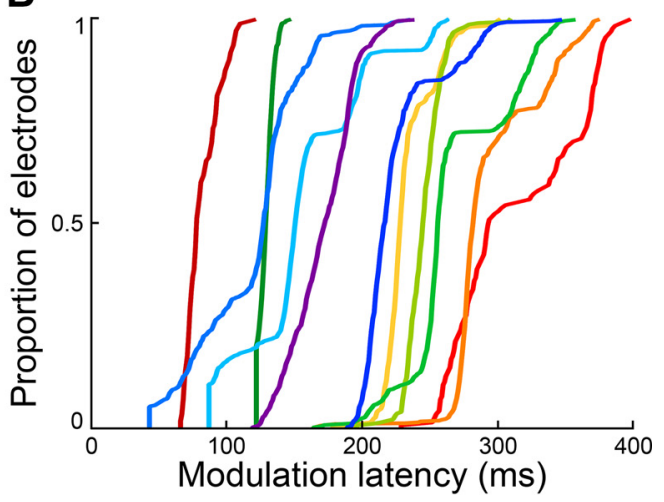

Figure 9. Attentional modulation latencies. $\boldsymbol{A}$, Mean modulation latency ( $\pm 95 \% \mathrm{Cl}$ ) by area. $N$ indicates the number of electrodes per area included in the analysis. $\boldsymbol{B}$, Cumulative distributions of modulation latencies for each area. Color-codes for areas are shown in $\boldsymbol{A}$.

cortical feedback modulation, they are consistent with the very fast attention latencies reported in LGN magnocellular populations (McAlonan et al., 2008), suggesting that a feedforward attentional modulation may be passed onto V1 from LGN. We also measured the response onset latencies of the suppression effects (Fig. 7C). In V1d/v, the suppression effect was even earlier than the fast component of the enhanced responses $(68 \pm 7 \mathrm{~ms}$, bootstrap randomization $p<0.01)$. The suppression effects in IPS3 sites with a negative MI were late $(265 \pm 142 \mathrm{~ms})$, on the order of the late enhancement effects found in IPS3 $(p=0.7)$.

Outside the topographic areas, parietal lobe sites had fast modulation latencies similar to those observed in IPS0 and IPS1/2 (124 $\pm 7 \mathrm{~ms})$, and temporal lobe sites had modulation latencies on the order of those in ventral extrastriate areas (223 \pm $16 \mathrm{~ms})$.

In a further test of the effect of attention on response onset latencies, we examined whether array onset responses were faster with attention. Previous studies of response onset latencies in extrastriate cortex of macaques had found a small, but consistent lag in response to ignored stimuli (Sundberg et al., 2012). However, we did not observe any systematic increases or lags in onset latencies with attention across the topographic areas $(t$ test, $p \sim 0.6$ ).

\section{Spatial tuning of response, memory, and attention fields}

Although our task was not designed to probe spatial tuning properties systematically and in detail (e.g., such as a function of eccentricity), we examined spatial tuning properties at a fixed peripheral eccentricity (i.e., $7^{\circ}$, which was the constant eccentricity at which the cue was presented) across the human visual system as well as outside of topographic visual cortex. Across all recording sites in each area that exhibited cue-evoked, spatiallytuned HFB response fields, we determined the population HFB spatial tuning curves (Fig. 10A), and the population widths at half-height of the tuning curves (Fig. 10B), as well as their individual distributions by area (Fig. 10C; see Materials and Methods for further details). We compared the tuning widths between the areas by generating bootstrapped distributions of mean tuning widths in each area after resampling, 500 times, from trials in each condition. The significance of the differences between these bootstrapped distributions was determined by applying the Hol$\mathrm{m}$-Bonferroni sequential correction for multiple comparisons at the target $\alpha$ level of $p<0.05$ (see Materials and Methods).

As expected from a wealth of fMRI studies in humans and electrophysiology studies in monkeys (Felleman and Van Essen,
1987; Dumoulin and Wandell, 2008; Wandell and Winawer, 2015), spatial tuning widths increased systematically across both the dorsal and ventral visual processing pathways (Fig. 10; Table 5). This progression was apparent in the population data (Fig. $10 B)$, as well as in the distribution of tuning widths from individual recording sites (Fig. 10C). Early visual areas V1-V3d/v had significantly narrower tuning widths (mean $=9.5 \pm 0.1^{\circ}, \mathrm{N}=$ 13) than dorsal extrastriate areas $(\mathrm{V} 3 \mathrm{~A} / \mathrm{B}$ and TO1-2 mean $=$ $13.1 \pm 0.2^{\circ}, N=12 ; p<0.05$; Table 5 shows all area-wise comparisons) and ventral extrastriate areas (LO1-2, hV4, and VO1-2 mean $\left.=13.2 \pm 0.4^{\circ}, N=11\right)$. Dorsal and ventral extrastriate areas were in turn more sharply tuned than posterior and anterior IPS areas (IPSO -2 mean $=15.5 \pm 0.5^{\circ}, N=12$; IPS3 -5 and SPL1 mean $\left.=15.8 \pm 0.6^{\circ}, N=11\right)$. Tuning widths of areas along the IPS were comparable. Nontopographic sites had tuning widths similar to higher-order topographic areas, with parietal lobe sites' tuning widths on the order of the topographic IPS sites (mean $=14.4 \pm 0.2^{\circ}, N=35$ ), and temporal lobe sites' widths comparable to the dorsal and ventral extrastriate sites (mean $=$ $11.9 \pm 0.7^{\circ}, N=14$ ).

We also determined the spatial tuning widths during the delay period (memory field) and in response to the array (attention field). At individual sites, a general broadening of the attention fields relative to the cue-evoked response fields was observed (Fig. 3B-E, purple compared with orange polar plots). At the population level, we investigated the effect of attention on the response field widths by examining the population of sites in each area that had a significant population enhancement effect (Fig. 7, sites from areas with a significant positive MI). We generated trial-wise bootstrapped distributions of mean memory and attention fields, from which we calculated the widths during the delay and in the late array window. We found that memory fields were significantly broader than response fields in TO1-2 and ventral extrastriate areas (increase of $2.7 \pm 0.3 \%$ and $7.5 \pm 0.5 \%$, respectively; bootstrap randomization test $p<0.001$ ), as well as the nontopographic areas (mean increase $=8.6 \pm 0.5 \%, p<0.01$ ) In contrast, we did not find significant differences in response and memory field widths in area V3A, nor the IPS areas IPS0-3 $(p \sim 0.1$; Fig. 11A).

All of the topographic areas that were significantly enhanced by attention (Fig. 7C, positive MI) showed increased attention field widths relative to their respective cue-evoked RFs (Fig. 11B). The effect was remarkably similar across the topographic areas, suggesting a global effect of attentional modulation on visual space, consistent with a recent fMRI study (Klein et al., 2014). 
A

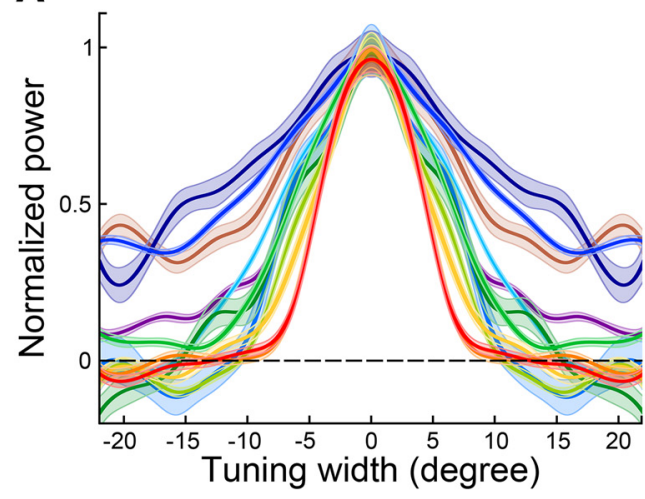

B

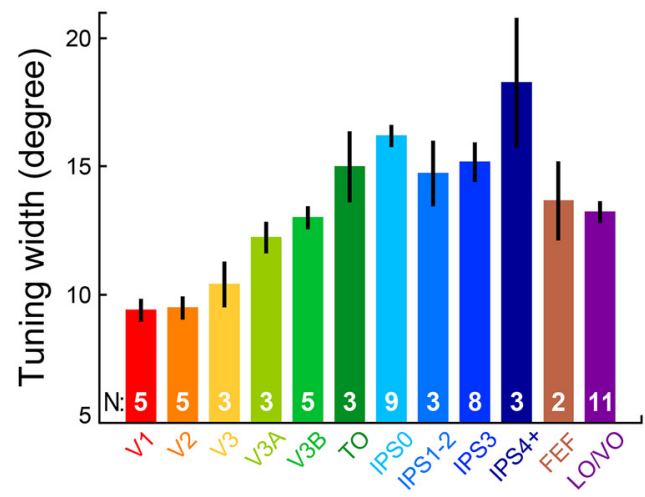

C

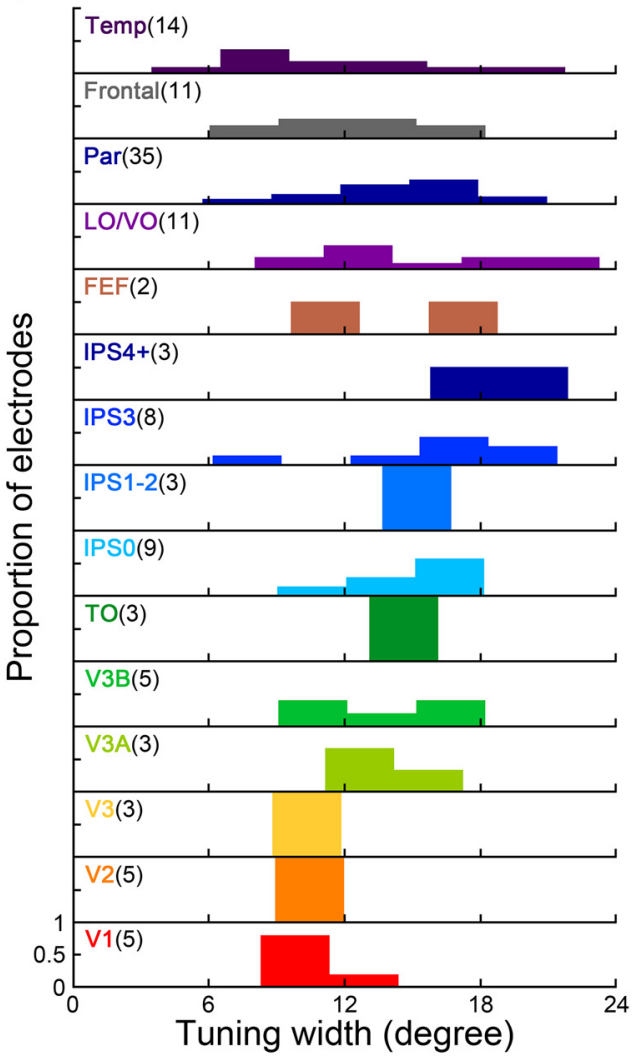

Figure 10. Spatial tuning widths of cue-evoked HFB response fields. $A$, Spatial tuning curves of cue-evoked mean HFB responses relative to baseline, normalized and centered on $\mathrm{RF}_{\mathrm{C}}$ and pooled across all sites with spatial RFs recorded within a given topographic area, $\pm 95 \% \mathrm{Cl}$. Color-codes for areas are shown in $\boldsymbol{B}$. B, Mean width of tuning curves $\pm 95 \%$ Cl. $N$ indicates the number of sites per area included in the analysis. $\boldsymbol{C}$, Tuning widths of sites with response fields by area. Number of sites per area is indicated in parentheses.

Table 5. Spatial tuning widths: significance by area

\begin{tabular}{|c|c|c|c|c|c|c|c|c|c|c|c|c|c|c|c|}
\hline & $\mathrm{V} 1 \mathrm{~d} / \mathrm{v}$ & $\mathrm{V} 2 \mathrm{~d} / \mathrm{v}$ & $\mathrm{V} 3 \mathrm{~d} / \mathrm{v}$ & V3A & V3B & T0 & IPSO & IPS1-2 & IPS3 & IPS4+ & FEF & LO/VO & Parietal & Frontal & Temporal \\
\hline $\mathrm{V} 1 \mathrm{~d} / \mathrm{v}$ & & & & - & - & • & - & & - & & & • & - & & • \\
\hline $\mathrm{V} 2 \mathrm{~d} / \mathrm{v}$ & & & & $\cdot$ & - & - & - & & - & & & • & - & & - \\
\hline $\mathrm{V} 3 \mathrm{~d} / \mathrm{v}$ & & & & - & $\cdot$ & - & $\cdot$ & & - & & & $\cdot$ & $\cdot$ & & $\cdot$ \\
\hline V3A & + & + & + & & - & & - & & & & & & $\cdot$ & & \\
\hline V3B & + & + & + & + & & & - & & & & & & - & & \\
\hline T0 & + & + & + & & & & & & & & + & & & + & \\
\hline IPSO & + & + & + & + & + & & & & & & & + & + & + & + \\
\hline IPS1-2 & & & & & & & & & - & & & & & & \\
\hline $\begin{array}{l}\text { IPS3 } \\
\text { IPS4+ }\end{array}$ & + & + & + & & & & & + & & & + & + & & + & + \\
\hline FEF & & & & & & - & & & - & & & & & + & \\
\hline LO/VO & + & + & + & & & & - & & • & & & & & & \\
\hline Parietal & + & + & + & + & + & & - & & & & & & & & \\
\hline Frontal & & & & & & - & - & & - & & - & & & & \\
\hline Temporal & + & + & + & & & & - & & • & & & & & & \\
\hline
\end{tabular}

Significance after Holm-Bonferroni sequential correction for multiple comparisons at target alpha level $p<0.05$. + Indicates that the tuning width of the column area was sharper than the row area. Indicates that the tuning width of the column area was broader than the row area. Blank cells indicate no significant difference between areas.

Except for areas $\mathrm{V} 1 \mathrm{~d} / \mathrm{v}$ and $\mathrm{V} 3 \mathrm{~d} / \mathrm{v}$, which had spatial attention field widths $\sim 8 \%$ broader than their response fields, all other topographic areas and the nontopographic sites showed broadening of spatial attention tuning widths on the order of $3 \%$ (mean increase $=2.6 \pm 0.3 \%$, all $p<0.001$ ). Such broadening may be due to expansion of RFs, as observed in single neurons when attention is allocated next to the RF (Anton-Erxleben et al., 2009). At the same time, there is also evidence that RFs shrink in extent when attention is allocated (Womelsdorf et al., 2006). Reconciling these contradictory observations with our findings may imply that, at the IFP spatial scale, the overall effect appears to be broadening of the IFP RF because of the many contributing individual neurons' RFs expanding and only a smaller number of individual neurons' RFs shrinking. Such broadening appears to occur only in response to visual stimuli, since we did not observe the same effect for memory fields. Relative to the memory field widths, attention fields were broader in areas TO1-2, IPS1-2, and IPS3 $(p<0.001)$, narrower in ventral extrastriate areas LO/VO and nontopographic areas $(p<0.001)$, and similar in V3A and IPS0 ( $p=0.4$; Fig. $11 C)$. 

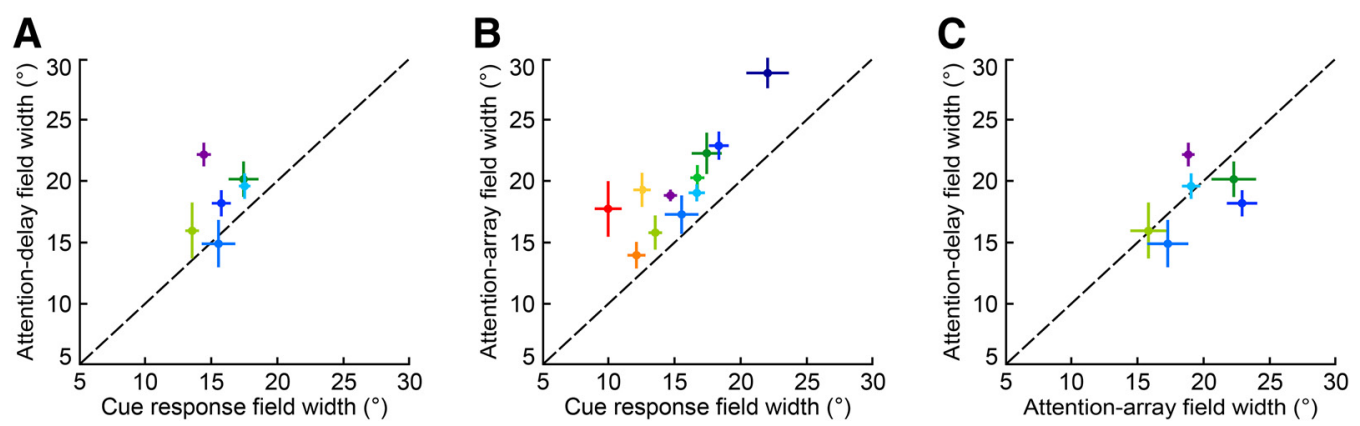

Figure 11. Cue-evoked RFs, memory, and attention fields. A, Mean field width of population tuning curves ( $\pm 95 \% \mathrm{Cl}$ ) for cue-evoked RFs versus memory fields for areas with a significant enhanced Ml in both windows. $\boldsymbol{B}$, Widths of cue-evoked RFs versus attention fields. $\boldsymbol{C}$, Widths of memory versus attention fields. Color-codes for areas are indicated in previous figures.

\section{Discussion}

We analyzed HFB responses from intracranial recordings of 626 electrodes implanted in 8 epilepsy patients, who performed a spatial attention task, to characterize a dynamic visual processing architecture, modulated by attentional task demands, in the human brain. Electrode locations were reconstructed using a probabilistic atlas of the human visual system (Wang et al., 2015). HFB responses showed high spatial selectivity and tuning, constituting ECoG RFs that were found within and outside the topographic visual system. Both RF widths and onset latencies increased systematically across the visual processing hierarchy. We used the spatial specificity of ECoG responses to quantitatively study spatial attention effects on baseline and visually-evoked activity. Attention effects were stronger, and attention modulation latencies were shorter, in extrastriate, and posterior parietal cortex than in early visual cortex. However, attention effects in anterior IPS and frontal cortex were weaker, and modulation latencies in anterior IPS were longer, than in posterior IPS. Together, the temporal dynamics and modulatory effects of spatial attention revealed in these studies only partially support attentional top-down models that assume a reversal of the visual processing hierarchy.

The electrophysiological basis of HFB responses is still an area of active investigation. HFB power fluctuations have been shown to correlate with multiunit activity from large populations of neurons in the vicinity of the recording electrode (Ray et al., 2008a; Ray and Maunsell, 2011; Rich and Wallis, 2017; Watson et al., 2018). More recent findings indicate CA + dendritic spikes in supragranular cortex as a principle contributor to pial HFB responses (Leszczyñski et al., Unpublished observations). However, models of HFB responses have also shown that power increases are predicted by increases in neuronal synchronization (Ray et al., 2008a). The underlying firing patterns may consist of multiple band-limited neuronal oscillations at different peak frequencies within the gamma band (Crone et al., 2011). Thus, it is possible that HFB responses index to some extent neuronal synchronization. We used the high spatial and temporal precision of HFB responses to track the temporal dynamics of visual and attentional processing.

\section{Spatial specificity of ECoG response fields}

Similar to previous reports from human early visual cortex (Yoshor et al., 2007; Winawer and Parvizi, 2016) and monkey visual cortex (Bosman et al., 2012), we found spatially confined ECoG RFs based on cue-evoked HFB responses. The spatial configurations of the RFs reflected the visual field representations of the underlying maps that are known from fMRI studies (Konen and Kastner, 2008; Silver and Kastner, 2009; Arcaro et al., 2011; Wang et al., 2015). Remarkably, electrodes that were located as little as $1 \mathrm{~cm}$ apart showed visual field sign reversals along the horizontal meridian with RF peaks in the upper and lower quadrants, respectively, underlining the impressive specificity of HFB responses, shown in several other domains (Crone et al., 1998; Canolty et al., 2007; Parvizi et al., 2012; Daitch et al., 2016). Interestingly, a large proportion of electrodes with ECoG RFs was found outside topographic cortex, equally distributed across the major lobes. The identification of spatially-selective, but relatively isolated sites outside of visual maps is difficult with techniques such as MEG/EEG and fMRI, which have a poor signal-to-noise ratio. Thus, spatially selective responses appear to be surprisingly ubiquitous outside of the topographic visual system.

\section{Temporal dynamics of feedforward processing}

This is the first report of systematic HFB response onset latencies across the human visual system (for LFP onset latencies, see Yoshor et al., 2007). Onset latencies increased gradually across the dorsal processing pathway, where we had systematic coverage, with estimated conduction delays of 10-15 ms between areas. Responses in $\mathrm{V} 1$ were recorded as fast as $50 \mathrm{~ms}$ after stimulus onset. In general, these results are in excellent agreement with monkey physiology studies (Schmolesky et al., 1998). Notably, we also found extremely short latencies in FEF that were comparable to the onset latencies in early visual cortex. However, a few of our findings were not predictable from what is known about the monkey visual system and therefore may be unique features of the human visual system. First, in humans, onset latencies in TO (the human MT/MST complex) were well $>100 \mathrm{~ms}$ and significantly longer than those in other dorsal extrastriate areas such as areas $\mathrm{V} 3 \mathrm{~d} / \mathrm{v}$, or $\mathrm{V} 3 \mathrm{~A}$. In contrast, in the monkey, onset latencies in these areas are typically shorter and similar to one another ( $70 \mathrm{~ms}$; Schmolesky et al., 1998; but see large range shown by Raiguel et al., 1989 and Azzopardi et al., 2003). Second, onset latencies between higher-order dorsal and ventral extrastriate areas, IPS1-4 and LO/VO were similar in humans. In contrast, onset latencies between dorsal and ventral higher-order cortex differ significantly in monkeys due to the relatively greater magnocellular input to the dorsal pathway. For example, neurons in LIP respond to shape stimuli with a latency of $\sim 60 \mathrm{~ms}$, whereas neurons in anterior inferotemporal cortex will respond after $\sim 100 \mathrm{~ms}$ (Lehky and Sereno, 2007). This discrepancy, as well as the longer latencies in $\mathrm{TO}$, may be attributable to the greater capacity of the human dorsal pathway to represent shape and object information (Konen and Kastner, 2008; Freud et al., 2016; Kastner et al., 2017). FMRI studies have shown that the human ventral and dorsal visual pathways represent nonspatial shape and object information similarly (Konen and Kastner, 2008), and thus the human dorsal pathway must receive a relatively greater input from the slower parvocellular system compared with the monkey dorsal pathway, which in turn might explain the longer onset latencies in TO and IPS. Despite these no- 
table human-specific features in the dynamics of feedforward processing, as indexed by response onset latencies, our results provide strong support for a hierarchical visual processing architecture in the human brain.

\section{Spatial attention effects and modulation latencies}

The temporal dynamics and strengths of attentional modulation have been interpreted as evidence in support of a top-down feedback model of selective attention. Specifically, monkey physiology studies have shown that attentional modulation latencies were shorter and the strength of attentional modulation was greater in higher-order cortex than in lower-order cortex. For example, Buffalo et al. (2010) recorded from areas V1, V2, and V4 and found that attention effects reversed modulation strengths and temporal order such that attentional enhancement was found to be larger and earlier in V4 and smaller and later in V1, with V2 showing intermediate results, similar to earlier findings by Mehta et al. (2000). These studies have provided support for the idea of a backward propagation of attentional feedback signals across the visual processing hierarchy.

We found widespread spatially-selective attention effects on HFB responses both on baseline activity during the delay and in response to the array, thereby corroborating previous ECoG studies on selective sensory processing (Ray et al., 2008b; Szczepanski et al., 2010; Davidesco et al., 2013; Zion Golumbic et al., 2013). In accordance with a large body of literature from monkey physiology (Luck et al., 1997; Cook and Maunsell, 2002) and human brain imaging (O'Connor et al., 2002; Siegel et al., 2008), attentional modulation was generally stronger in higher-order compared with lower-order areas.

Specifically, our recordings focused on a multitude of areas along the dorsal processing pathway. We found indeed a systematic "backward propagation" in early visual cortex, from areas V3 to V2 and V1 with increasingly longer attentional modulation latencies, and these latencies were also significantly longer than those obtained in dorsal extrastriate cortex. However, the temporal dynamics in dorsal extrastriate and posterior parietal cortex were more complex. For example, area TO and IPS0 had significantly faster latencies than IPS3. Thus, these modulation latencies did not appear to follow a strictly hierarchical processing that was reversed during spatial attention and they do not lend unequivocal support for the top-down feedback model. However, our assumptions on the visual processing hierarchy along the human dorsal pathway can only be tentative. Based on the anatomical locations of areas, one would assume that TO projects to and receives feedback from the IPS areas, and the same would hold for the posterior relative to the anterior IPS areas, but detailed anatomical studies on structural connectivity are lacking. Connectivity, both structurally and functionally, may be increasingly more divergent in higher-order cortex, thereby promoting parallel rather than hierarchical processing. For example, anterior IPS shows grip- and reach-related activations (Konen et al., 2013) as well as representations of tool and manipulable object information (Mruczek et al., 2013). Further, posterior IPS, but not IPS3 -5 , has been reported to interact with other frontoparietal attention areas, like FEF and supplementary eye field, in visuospatial attention tasks (Szczepanski et al., 2013). Anterior IPS may thus contribute to a different network than posterior IPS, which may predominantly serve visuospatial attention and oculomotor functions. Interestingly, based on analyses of directed feedforward and feedback signaling indexed by synchronization in certain frequency channels, Michalareas et al. (2016) placed the anterior IPS areas below the posterior IPS areas in their functional hierarchy, which is further evidence for the more complex inter-areal dynamics during attentional processing particularly in human parietal cortex. Further, it is noteworthy that cortical network interactions are influenced by additional sources such as thalamic nuclei, which complicates the interpretation of temporal corticocortical interactions (for an extensive discussion of alternative attention control models, see Halassa and Kastner, 2017).

\section{Attentional modulation in V1}

Attention effects on array-evoked activity were moderate in early visual cortex. Both enhancement and suppression effects were found in V1, without a net effect of attention. The strongest attention effect that we obtained in V1 was attentional suppression, likely due to modulation of activity in extra-RF surrounds. These findings are consistent with previous monkey physiology studies that have shown attention-related decreases in LFP gamma power in the $40-60 \mathrm{~Hz}$ frequency band and spike-field coherence in V1 using stimuli that engaged suppressive extra-RF surrounds (Chalk et al., 2010), as well as with findings of attention-related increases of LFP gamma power when extra-RF surrounds were less stimulated (Bosman et al., 2012). Thus, it is possible that HFB responses also reflect neuronal synchronization processes, because attention-related modulation of spiking activity is typically moderate (Motter, 1993; Luck et al., 1997; McAdams and Maunsell, 1999; Grunewald et al., 2002; Marcus and Van Essen, 2002; Yoshor et al., 2007).

Interestingly, we also found evidence of attentional feedforward modulation in V1, where three modulatory temporal components were found, two early components that were observed at array onset of attentional suppression and enhancement, and a late component that was observed with attentional enhancement and followed the top-down feedback model, discussed above. In monkey physiology studies, attentional feedforward modulation has been found in LGN and thalamic reticular nucleus (TRN; McAlonan et al., 2008). This modulation may be mediated through direct influences of prefrontal cortex on the TRN that bypass corticocortical feedback, as shown in the mouse model (Wimmer et al., 2015). The feedforward modulation observed in LGN-TRN may be passed on to V1 and thus account for our observations. In human EEG studies, attention effects on the earliest component (the "C1"; $\sim 50 \mathrm{~ms}$ onset) that is typically attributed to a generator in striate cortex have been controversial (Martínez et al., 1999; Di Russo et al., 2003; Kelly et al., 2008). Our findings of two early components support the possibility that the earliest EEG component may be modulated by spatial attention.

\section{References}

Angelucci A, Levitt JB, Walton EJ, Hupe JM, Bullier J, Lund JS (2002) Circuits for local and global signal integration in primary visual cortex. J Neurosci 22:8633-8646. CrossRef Medline

Anton-Erxleben K, Stephan VM, Treue S (2009) Attention reshapes centersurround receptive field structure in macaque cortical area MT. Cereb Cortex 19:2466-2478. CrossRef Medline

Arcaro MJ, Pinsk MA, Li X, Kastner S (2011) Visuotopic organization of macaque posterior parietal cortex: a functional magnetic resonance imaging study. J Neurosci 31:2064-2078. CrossRef Medline

Azzopardi P, Fallah M, Gross CG, Rodman HR (2003) Response latencies of neurons in visual areas MT and MST of monkeys with striate cortex lesions. Neuropsychologia 41:1738-1756. CrossRef Medline

Bair W, Cavanaugh JR, Movshon JA (2003) Time course and time-distance relationships for surround suppression in macaque V1 neurons. J Neurosci 23:7690-7701. CrossRef Medline

Barceló F, Suwazono S, Knight RT (2000) Prefrontal modulation of visual processing in humans. Nat Neurosci 3:399-403. CrossRef Medline

Bastos AM, Vezoli J, Bosman CA, Schoffelen JM, Oostenveld R, Dowdall JR, De Weerd P, Kennedy H, Fries P (2015) Visual areas exert feedforward and feedback influences through distinct frequency channels. Neuron 85:390-401. CrossRef Medline 
Bosman CA, Schoffelen JM, Brunet N, Oostenveld R, Bastos AM, Womelsdorf T, Rubehn B, Stieglitz T, De Weerd P, Fries P (2012) Attentional stimulus selection through selective synchronization between monkey visual areas. Neuron 75:875-888. CrossRef Medline

Buffalo EA, Fries P, Landman R, Liang H, Desimone R (2010) A backward progression of attentional effects in the ventral stream. Proc Natl Acad Sci U S A 107:361-365. CrossRef Medline

Buschman TJ, Kastner S (2015) From behavior to neural dynamics: an integrated theory of attention. Neuron 88:127-144. CrossRef Medline

Buzsáki G, Anastassiou CA, Koch C (2012) The origin of extracellular fields and currents: EEG, ECoG, LFP and spikes. Nat Rev Neurosci 13:407-420. CrossRef Medline

Canolty RT, Soltani M, Dalal SS, Edwards E, Dronkers NF, Nagarajan SS, Kirsch HE, Barbaro NM, Knight RT (2007) Spatiotemporal dynamics of word processing in the human brain. Front Neurosci 1:185-196. CrossRef Medline

Caspari N, Janssens T, Mantini D, Vandenberghe R, Vanduffel W (2015) Covert shifts of spatial attention in the macaque monkey. J Neurosci 35:7695-7714. CrossRef Medline

Chalk M, Herrero JL, Gieselmann MA, Delicato LS, Gotthardt S, Thiele A (2010) Attention reduces stimulus-driven gamma frequency oscillations and spike field coherence in V1. Neuron 66:114-125. CrossRef Medline

Cheung C, Hamilton LS, Johnson K, Chang EF (2016) The auditory representation of speech sounds in human motor cortex. eLife 5:e12577. CrossRef Medline

Cook EP, Maunsell JH (2002) Attentional modulation of behavioral performance and neuronal responses in middle temporal and ventral intraparietal areas of macaque monkey. J Neurosci 22:1994-2004. CrossRef Medline

Corbetta M, Shulman GL (2002) Control of goal-directed and stimulusdriven attention in the brain. Nat Rev Neurosci 3:201-215. CrossRef Medline

Corbetta M, Shulman GL (2011) Spatial neglect and attention networks. Annu Rev Neurosci 34:569-599. CrossRef Medline

Cox MA, Dougherty K, Adams GK, Reavis EA, Westerberg JA, Moore BS, Leopold DA, Maier A (2017) Spiking suppression precedes cued attentional enhancement of neural responses in primary visual cortex. Cereb Cortex. Advance online publication. CrossRef Medline

Crone NE, Miglioretti DL, Gordon B, Lesser RP (1998) Functional mapping of human sensorimotor cortex with electrocorticographic spectral analysis: II. Event-related synchronization in the gamma band. Brain 121: 2301-2315. CrossRef Medline

Crone NE, Sinai A, Korzeniewska A (2006) High-frequency gamma oscillations and human brain mapping with electrocorticography. Prog Brain Res 159:275-295. CrossRef Medline

Crone NE, Korzeniewska A, Franaszczuk PJ (2011) Cortical gamma responses: searching high and low. Int J Psychophysiol 79:9-15. CrossRef Medline

Daitch AL, Foster BL, Schrouff J, Rangarajan V, Kaşikçi I, Gattas S, Parvizi J (2016) Mapping human temporal and parietal neuronal population activity and functional coupling during mathematical cognition. Proc Natl Acad Sci U S A 113:E7277-E7286. CrossRef Medline

Dale AM, Fischl B, Sereno MI (1999) Cortical surface-based analysis: I. Segmentation and surface reconstruction. Neuroimage 9:179-194. CrossRef Medline

Davidesco I, Harel M, Ramot M, Kramer U, Kipervasser S, Andelman F, Neufeld MY, Goelman G, Fried I, Malach R (2013) Spatial and objectbased attention modulates broadband high-frequency responses across the human visual cortical hierarchy. J Neurosci 33:1228-1240. CrossRef Medline

Delorme A, Makeig S (2004) EEGLAB: an open source toolbox for analysis of single-trial EEG dynamics including independent component analysis. J Neurosci Methods 134:9-21. CrossRef Medline

Desikan RS, Ségonne F, Fischl B, Quinn BT, Dickerson BC, Blacker D, Buckner RL, Dale AM, Maguire RP, Hyman BT, Albert MS, Killiany RJ (2006) An automated labeling system for subdividing the human cerebral cortex on MRI scans into gyral based regions of interest. Neuroimage 31:968980. CrossRef Medline

Desimone R, Duncan J (1995) Neural mechanisms of selective visual attention. Annu Rev Neurosci 18:193-222. CrossRef Medline

Di Russo F, Martínez A, Hillyard SA (2003) Source analysis of event-related cortical activity during visuo-spatial attention. Cereb Cortex 13:486-499. CrossRef Medline

Dumoulin SO, Wandell BA (2008) Population receptive field estimates in human visual cortex. Neuroimage 39:647-660. CrossRef Medline

Engel AK, Fries P (2010) Beta-band oscillations: signalling the status quo? Curr Opin Neurobiol 20:156-165. CrossRef Medline

Eriksen BA, Eriksen CW (1974) Effects of noise letters upon the identification of a target letter in a nonsearch task. Percept Psychophys 16:143-149. CrossRef

Eriksen CW (1995) The flankers task and response competition: a useful tool for investigating a variety of cognitive problems. Vis Cogn 2:101-118. CrossRef

Felleman DJ, Van Essen DC (1987) Receptive field properties of neurons in area V3 of macaque monkey extrastriate cortex. J Neurophysiol 57:889 920. CrossRef Medline

Felleman DJ, Van Essen DC (1991) Distributed hierarchical processing in the primate cerebral cortex. Cereb Cortex 1:1-47. CrossRef Medline

Fischl B, Sereno MI, Dale AM (1999) Cortical surface-based analysis: II. Inflation, flattening, and a surface-based coordinate system. Neuroimage 9:195-207. CrossRef Medline

Flinker A, Chang EF, Barbaro NM, Berger MS, Knight RT (2011) Subcentimeter language organization in the human temporal lobe. Brain Lang 117:103-109. CrossRef Medline

Freud E, Plaut DC, Behrmann M (2016) "What" is happening in the dorsal visual pathway. Trends Cogn Sci 20:773-784. CrossRef Medline

Fries P (2009) Neuronal gamma-band synchronization as a fundamental process in cortical computation. Annu Rev Neurosci 32:209-224. CrossRef Medline

Gregoriou GG, Gotts SJ, Zhou H, Desimone R (2009) High-frequency, long-range coupling between prefrontal and visual cortex during attention. Science 324:1207-1210. CrossRef Medline

Grunewald A, Bradley DC, Andersen RA (2002) Neural correlates of structure-from-motion perception in macaque V1 and MT. J Neurosci 22:6195-6207. CrossRef Medline

Halassa MM, Kastner S (2017) Thalamic functions in distributed cognitive control. Nat Neurosci 20:1669-1679. CrossRef Medline

Hermes D, Miller KJ, Vansteensel MJ, Aarnoutse EJ, Leijten FS, Ramsey NF (2012) Neurophysiologic correlates of fMRI in human motor cortex. Hum Brain Mapp 33:1689-1699. CrossRef Medline

Ito M, Gilbert CD (1999) Attention modulates contextual influences in the primary visual cortex of alert monkeys. Neuron 22:593-604. CrossRef Medline

Kastner S, Ungerleider LG (2000) Mechanisms of visual attention in the human cortex. Annu Rev Neurosci 23:315-341. CrossRef Medline

Kastner S, Chen Q, Jeong SK, Mruczek REB (2017) A brief comparative review of primate posterior parietal cortex: a novel hypothesis on the human toolmaker. Neuropsychologia 105:123-134. CrossRef Medline

Kelly SP, Gomez-Ramirez M, Foxe JJ (2008) Spatial attention modulates initial afferent activity in human primary visual cortex. Cereb Cortex 18:2629-2636. CrossRef Medline

Klein BP, Harvey BM, Dumoulin SO (2014) Attraction of position preference by spatial attention throughout human visual cortex. Neuron 84: 227-237. CrossRef Medline

Konen CS, Kastner S (2008) Representation of eye movements and stimulus motion in topographically organized areas of human posterior parietal cortex. J Neurosci 28:8361-8375. CrossRef Medline

Konen CS, Mruczek RE, Montoya JL, Kastner S (2013) Functional organization of human posterior parietal cortex: grasping-and reaching-related activations relative to topographically organized cortex. J Neurophysiol 109:2897-2908. CrossRef Medline

Kreiman G, Hung CP, Kraskov A, Quiroga RQ, Poggio T, DiCarlo JJ (2006) Object selectivity of local field potentials and spikes in the macaque inferior temporal cortex. Neuron 49:433-445. CrossRef Medline

Lachaux JP, George N, Tallon-Baudry C, Martinerie J, Hugueville L, Minotti L, Kahane P, Renault B (2005) The many faces of the gamma band response to complex visual stimuli. Neuroimage 25:491-501. CrossRef Medline

Lee J, Williford T, Maunsell JH (2007) Spatial attention and the latency of neuronal responses in macaque area V4. J Neurosci 27:9632-9637. CrossRef Medline

Lehky SR, Sereno AB (2007) Comparison of shape encoding in primate dor- 
sal and ventral visual pathways. J Neurophysiol 97:307-319. CrossRef Medline

Lisman JE, Jensen O (2013) The theta-gamma neural code. Neuron 77: 1002-1016. CrossRef Medline

Luck SJ, Girelli M, McDermott MT, Ford MA (1997) Bridging the gap between monkey neurophysiology and human perception: an ambiguity resolution theory of visual selective attention. Cogn Psychol 33:64-87. CrossRef Medline

Marcus DS, Van Essen DC (2002) Scene segmentation and attention in primate cortical areas V1 and V2. J Neurophysiol 88:2648-2658. CrossRef Medline

Maris E, Oostenveld R (2007) Nonparametric statistical testing of EEG-and MEG-data. J Neurosci Methods 164:177-190. CrossRef Medline

Markov NT, Vezoli J, Chameau P, Falchier A, Quilodran R, Huissoud C, Lamy C, Misery P, Giroud P, Ullman S, Barone P, Dehay C, Knoblauch K, Kennedy H. (2014) Anatomy of hierarchy: feedforward and feedback pathways in macaque visual cortex. J Comp Neurol 522:225-259. CrossRef Medline

Martínez A, Anllo-Vento L, Sereno MI, Frank LR, Buxton RB, Dubowitz DJ, Wong EC, Hinrichs H, Heinze HJ, Hillyard SA.zens(1999) Involvement of striate and extrastriate visual cortical areas in spatial attention. Nat Neurosci 2:364-369. CrossRef Medline

McAdams CJ, Maunsell JH (1999) Effects of attention on the reliability of individual neurons in monkey visual cortex. Neuron 23:765-773. CrossRef Medline

McAlonan K, Cavanaugh J, Wurtz RH (2008) Guarding the gateway to cortex with attention in visual thalamus. Nature 456:391-394. CrossRef Medline

Mehta AD, Ulbert I, Schroeder CE (2000) Intermodal selective attention in monkeys: I. Distribution and timing of effects across visual areas. Cereb Cortex 10:343-358. CrossRef Medline

Mesgarani N, Cheung C, Johnson K, Chang EF (2014) Phonetic feature encoding in human superior temporal gyrus. Science 343:1006-1010. CrossRef Medline

Michalareas G, Vezoli J, van Pelt S, Schoffelen JM, Kennedy H, Fries P (2016) Alpha-beta and gamma rhythms subserve feedback and feedforward influences among human visual cortical areas. Neuron 89:384-397. CrossRef Medline

Moore T, Armstrong KM (2003) Selective gating of visual signals by microstimulation of frontal cortex. Nature 421:370-373. CrossRef Medline

Moore T, Zirnsak M (2017) Neural mechanisms of selective visual attention. Annu Rev Psychol 68:47-72. CrossRef Medline

Motter BC (1993) Focal attention produces spatially selective processing in visual cortical areas V1, V2, and V4 in the presence of competing stimuli. J Neurophysiol 70:909-919. CrossRef Medline

Mruczek RE, von Loga IS, Kastner S (2013) The representation of tool and non-tool object information in the human intraparietal sulcus. J Neurophysiol 109:2883-2896. CrossRef Medline

O'Connor DH, Fukui MM, Pinsk MA, Kastner S (2002) Attention modulates responses in the human lateral geniculate nucleus. Nat Neurosci 5:1203-1209. CrossRef Medline

Ozeki H, Finn IM, Schaffer ES, Miller KD, Ferster D (2009) Inhibitory stabilization of the cortical network underlies visual surround suppression. Neuron 62:578-592. CrossRef Medline

Parvizi J, Kastner S (2018) Promises and limitations of human intracranial electroencephalography. Nat Neurosci 21:474-483. CrossRef Medline

Parvizi J, Jacques C, Foster BL, Witthoft N, Rangarajan V, Weiner KS, GrillSpector K (2012) Electrical stimulation of human fusiform faceselective regions distorts face perception. J Neurosci 32:14915-14920. CrossRef Medline

Raiguel SE, Lagae L, Gulyàs B, Orban GA (1989) Response latencies of visual cells in macaque areas V1, V2 and V5. Brain Res 493:155-159. CrossRef Medline

Ray S, Crone NE, Niebur E, Franaszczuk PJ, Hsiao SS (2008a) Neural correlates of high-gamma oscillations $(60-200 \mathrm{~Hz})$ in macaque local field potentials and their potential implications in electrocorticography. J Neurosci 28:11526-11536. CrossRef Medline

Ray S, Niebur E, Hsiao SS, Sinai A, Crone NE (2008b) High-frequency gamma activity $(80-150 \mathrm{~Hz})$ is increased in human cortex during selective attention. Clin Neurophysiol 119:116-133. CrossRef Medline

Ray S, Maunsell JH (2011) Different origins of gamma rhythm and highgamma activity in macaque visual cortex. PLoS Biol 9:e1000610. CrossRef Medline
Reynolds JH, Chelazzi L (2004) Attentional modulation of visual processing. Annu Rev Neurosci 27:611-647. CrossRef Medline

Rich EL, Wallis JD (2017) Spatiotemporal dynamics of information encoding revealed in orbitofrontal high-gamma. Nat Commun 8:1139. CrossRef Medline

Saad ZS, Reynolds RC, Argall B, Japee S, Cox RW (2004) SUMA: an interface for surface-based intra-and inter-subject analysis with AFNI. IEEE International Symposium on Biomedical Imaging: Nano to Macro, pp 1510-1513, Arlington, VA.

Saalmann YB, Kastner S (2011) Cognitive and perceptual functions of the visual thalamus. Neuron 71:209-223. CrossRef Medline

Saalmann YB, Pinsk MA, Wang L, Li X, Kastner S (2012) The pulvinar regulates information transmission between cortical areas based on attention demands. Science 337:753-756. CrossRef Medline

Schmolesky MT, Wang Y, Hanes DP, Thompson KG, Leutgeb S, Schall JD, Leventhal AG (1998) Signal timing across the macaque visual system. J Neurophysiol 79:3272-3278. CrossRef Medline

Siegel M, Donner TH, Oostenveld R, Fries P, Engel AK (2008) Neuronal synchronization along the dorsal visual pathway reflects the focus of spatial attention. Neuron 60:709-719. CrossRef Medline

Silver MA, Kastner S (2009) Topographic maps in human frontal and parietal cortex. Trends Cogn Sci 13:488-495. CrossRef Medline

Sundberg KA, Mitchell JF, Gawne TJ, Reynolds JH (2012) Attention influences single unit and local field potential response latencies in visual cortical area V4. J Neurosci 32:16040-16050. CrossRef Medline

Szczepanski SM, Konen CS, Kastner S (2010) Mechanisms of spatial attention control in frontal and parietal cortex. J Neurosci 30:148-160. CrossRef Medline

Szczepanski SM, Pinsk MA, Douglas MM, Kastner S, Saalmann YB (2013) Functional and structural architecture of the human dorsal frontoparietal attention network. Proc Natl Acad Sci U SA 110:15806-15811. CrossRef Medline

Szczepanski SM, Crone NE, Kuperman RA, Auguste KI, Parvizi J, Knight RT (2014) Dynamic changes in phase-amplitude coupling facilitate spatial attention control in fronto-parietal cortex. PLoS Biol 12:e1001936. CrossRef Medline

van Kerkoerle T, Self MW, Dagnino B, Gariel-Mathis MA, Poort J, van Der Togt C, Roelfsema PR (2014) Alpha and gamma oscillations characterize feedback and feedforward processing in monkey visual cortex. Proc Natl Acad Sci U S A 111:14332-14341. CrossRef Medline

Voytek B, D'Esposito M, Crone N, Knight RT (2013) A method for eventrelated phase/amplitude coupling. Neuroimage 64:416-424. CrossRef Medline

Wandell BA, Winawer J (2015) Computational neuroimaging and population receptive fields. Trends Cogn Sci 19:349-357. CrossRef Medline

Wang L, Mruczek RE, Arcaro MJ, Kastner S (2015) Probabilistic maps of visual topography in human cortex. Cereb Cortex 25:3911-3931. CrossRef Medline

Watson BO, Ding M, Buzsáki G (2018) Temporal coupling of field potentials and action potentials in the neocortex. Eur J Neurosci 48:2482-2497. CrossRef Medline

Wimmer RD, Schmitt LI, Davidson TJ, Nakajima M, Deisseroth K, Halassa MM (2015) Thalamic control of sensory selection in divided attention. Nature 526:705-709. CrossRef Medline

Winawer J, Parvizi J (2016) Linking electrical stimulation of human primary visual cortex, size of affected cortical area, neuronal responses, and subjective experience. Neuron 92:1213-1219. CrossRef Medline

Womelsdorf T, Anton-Erxleben K, Pieper F, Treue S (2006) Dynamic shifts of visual receptive fields in cortical area MT by spatial attention. Nat Neurosci 9:1156-1160. CrossRef Medline

Yoshor D, Ghose GM, Bosking WH, Sun P, Maunsell JH (2007) Spatial attention does not strongly modulate neuronal responses in early human visual cortex. J Neurosci 27:13205-13209. CrossRef Medline

Zhang S, Xu M, Kamigaki T, Hoang Do JP, Chang WC, Jenvay S, Miyamichi K, Luo L, Dan Y (2014) Long-range and local circuits for top-down modulation of visual cortex processing. Science 345:660-665. CrossRef Medline

Zion Golumbic EM, Ding N, Bickel S, Lakatos P, Schevon CA, McKhann GM, Goodman RR, Emerson R, Mehta AD, Simon JZ, Poeppel D, Schroeder CE (2013) Mechanisms underlying selective neuronal tracking of attended speech at a "cocktail party". Neuron 77:980-991. CrossRef Medline 